\title{
Statistical and superposed epoch study of dipolarization events using data from Wind perigee passes
}

\author{
K. Sigsbee ${ }^{1}$, J. A. Slavin ${ }^{2}$, R. P. Lepping ${ }^{2}$, A. Szabo ${ }^{2}$, M. Øieroset ${ }^{3}$, M. L. Kaiser ${ }^{2}$, M. J. Reiner ${ }^{4}$, and H. J. Singer ${ }^{5}$ \\ ${ }^{1}$ Department of Physics and Astronomy, University of Iowa, Iowa City, Iowa, 52242 USA \\ ${ }^{2}$ Laboratory for Extraterrestrial Physics, NASA Goddard Space Flight Center, Greenbelt, Maryland 20771, USA \\ ${ }^{3}$ Space Sciences Laboratory, University of California, Berkeley, California, USA \\ ${ }^{4}$ The Catholic University of America, Washington, D.C., 20064, USA \\ ${ }^{5}$ NOAA Space Environment Center, Boulder, Colorado, 80305 USA
}

Received: 5 August 2004 - Revised: 23 November 2004 - Accepted: 15 December 2004 - Published: 30 March 2005

\begin{abstract}
From 1995 to 2000, the Wind spacecraft spent over $500 \mathrm{~h}$ in the magnetotail, much of it within $\sim 2 \times 10^{4} \mathrm{~km}$ of the predicted location of the neutral sheet. Wind passed through the near magnetotail at distances of $-15 R_{E}<X$ $\mathrm{GSM}<-6 R_{E}$ on 35 occasions. Another 10 passes took place at distances of $-30 R_{E}<X$ GSM $<-15 R_{E}$. We identified 65 dipolarization events in the Wind magnetic field data set between $Y$ GSM $\sim-16$ and $+16 R_{E}$ based upon our requirements that the magnetic field inclination had to change by more than $15^{\circ}$, the maximum inclination angle had to be greater than $20^{\circ}$, and the inclination angle had to increase by a factor of at least 1.5. Most of the dipolarization events occurred in the pre-midnight region of the magnetotail and were accompanied by earthward flows with speeds greater than $100 \mathrm{~km} / \mathrm{s}$. The properties of the dipolarization events did not depend upon the $Y$ GSM position. However, they did vary with the distance to the neutral sheet. Isolated dipolarization events, defined as occurring more than 20 min apart, were characterized by a decrease in $B_{x}$ GSM and $B_{T O T A L}$, and an increase in $B_{z}$ GSM and the magnetic field inclination. Dipolarizations that occurred as part of a series of small dipolarizations spaced less than $20 \mathrm{~min}$ apart were characterized by a transient increase in $B_{z}$ GSM and the magnetic field inclination, but no significant change in $B_{x}$ GSM and $B_{\text {TOTAL}}$. The events consisting of a series of small dipolarizations occurred predominantly near midnight. We interpret these results in terms of two different modes of magnetotail convection: 1) a classical substorm pattern featuring storage of magnetic energy in the tail lobes which is explosively released at onset, and 2) a directly driven process.
\end{abstract}

Keywords. Magnetospheric physics (Storms and substorms; Magnetotail; Magnetospheric configuration and dynamics)

Correspondence to: K. Sigsbee

(kms@beta.physics.uiowa.edu)

\section{Introduction}

Substorms result from a complex set of interactions between the ionosphere, the magnetosphere, and the solar wind that usually involve the storage of energy and magnetic flux in the tail lobes and its explosive release at the onset of the substorm expansion phase. The signatures observed by satellites in various regions of the night-side magnetosphere during a typical substorm include bursts of high-speed earthward and tailward plasma flow, magnetic field dipolarizations, lowfrequency fluctuations of the electric and magnetic fields, the ejection of plasmoids, and energetic particle injections at geosynchronous orbit (e.g. Slavin et al., 2002; Nagai, 1982). Recent theories have suggested that the observations of dipolarizations and earthward flows in the magnetotail may be related to the formation of the substorm current wedge and the generation of the Pi2 pulsations observed by geosynchronous satellites and ground magnetometer stations (Shiokawa et al., 1998; Kepko and Kivelson, 1999, 2001). In this paper, we will use data from passes of the Wind spacecraft through the magnetotail to study the extent of the region where magnetic field dipolarizations are observed. We will also examine the properties of dipolarization events as a function of location and compare the characteristics of isolated dipolarizations and dipolarizations that occurred as part of a series of small, closely spaced dipolarizations.

Investigations of the effects of earthward flow bursts in the magnetotail during substorms by Hesse and Birn (1991) and Shiokawa et al. $(1997,1998)$ have suggested that the braking of high-speed earthward flows launched by reconnection at a near-Earth neutral line may be responsible for the generation of the substorm current systems and the Pi2 pulsations observed by satellites at geosynchronous orbit and by ground magnetometer stations at substorm onset. According to this picture of substorms, the high-speed flows launched by magnetic reconnection at the near-Earth neutral line (NENL) carry large amounts of magnetic flux earthward, which eventually starts to pile up in the near magnetotail region where the flows "brake" as they compress the inner magnetosphere. 
This pile-up of magnetic flux is observed by spacecraft in the magnetotail as a magnetic field dipolarization. During dipolarization, the magnetotail magnetic field undergoes a change from a tail-like magnetic field configuration to a more dipolar magnetic field configuration. As defined by Shiokawa et al. (1997, 1998), the braking point of high-speed earthward flows is the boundary between the plasma sheet and dipolar magnetic field regions at the inner edge of the neutral sheet. The deceleration of earthward flows in the magnetotail generates field-aligned currents through the pressure gradients and the divergence of the dawnward inertial current. In a simulation performed by Birn et al. (1999) the flows were stopped over a distance of several $R_{E}$ and it was found that the contribution of the inertial current, which results from the coupling of the perpendicular fluid flow to the magnetic field, is almost an order of magnitude smaller than the currents caused by pressure gradients. The currents generated by the pressure gradients respond to distortions of the magnetic field and persist even after the flows cease. According to Shiokawa et al. (1998), Pi2 pulsations are also produced as the flows are decelerated, pressure gradients are created, and currents are generated.

Many studies have attempted to characterize magnetic field dipolarizations from geosynchronous orbit out to radial distances near $30 R_{E}$. Data from the GOES family of geosynchronous spacecraft have shown that the magnetic field configuration at geosynchronous orbit becomes more tail-like before substorm onset (Nagai, 1982, 1991). This change is thought to be caused by an intensification and/or an inward motion of the cross-tail current associated with the storage of energy in the magnetotail during the substorm growth phase (Kokubun and McPherron, 1981). At the onset of the substorm expansion phase, the dipolarization at geosynchronous orbit begins in a narrow region centered on 23:30 LT (Nagai, 1991). As shown by Nagai (1982), the increase in the $H$ component of the magnetic field (parallel to the Earth's dipole axis and positive northward) associated with dipolarization begins simultaneously with the substorm onset recorded by ground magnetometer data in the pre-midnight region. However, at local times more than $1 \mathrm{~h}$ away from the initial dipolarization onset, the magnetic field at geosynchronous orbit can still continue to become more tail-like until the dipolarization expands westward and eastward. According to Nagai (1991), it takes $11 \mathrm{~min}$ for the dipolarization to expand eastward to 03:00 MLT, and $15 \mathrm{~min}$ for it to expand westward to 20:00 MLT.

A detailed study of magnetic field dipolarization events at larger radial distances was performed by Lopez et al. (1988) using magnetic field data from the AMPTE/CCE spacecraft, which had an apogee of $8.8 R_{E}$. In this study, 103 dipolarization events were identified from March to August 1985 when $a \geq 15^{\circ}$ change in magnetic field inclination to the equatorial plane marked the transition from a tail-like field configuration to a more dipolar configuration. No dipolarization events were observed by AMPTE/CCE for geocentric radii $\leq 6.4 R_{E}$, suggesting that geosynchronous spacecraft may sometimes be located too far earthward to observe dipolarization events. Lopez et al. (1988) found that the number of dipolarization events observed by AMTPE/CCE increased with increasing radial distance from Earth and that the total magnetic field decreased during most of the AMTPE/CCE dipolarization events. The dipolarization events studied by Lopez et al. (1988) were distributed from 19.9 to 4.7 MLT, with a peak near midnight, which roughly corresponds to the region in which substorm activity and the substorm current wedge are thought to be initiated. Lopez et al. (1988) found the occurrence frequency of dipolarization events was 4 times greater for $K_{p} \geq 4+$ than it was for $K_{p} \leq 3-$ and that the central meridian of the substorm current wedge shifts from pre-midnight to post-midnight as the intensity of geomagnetic activity increases.

More recently, Baumjohann et al. (1999) performed a superposed epoch study of dipolarization events using Geotail data from $11 \leq R \leq 31 R_{E}$ and $0 \leq Y \mathrm{GSM} \leq 10 R_{E}$. They found 66 dipolarizations in the Geotail data from 1995 to 1997 for which data from mid-latitude ground stations were also available to aid in the determination of the substorm onset times. All of the events studied were from the central plasma sheet and were accompanied by high-speed earthward flows. Baumjohann et al. (1999) examined the average properties of the magnetic field during dipolarization in $5 R_{E}$ bins of radial distance and found that, on average, the magnetic field inclination to the equatorial plane increased by a factor of 2-3 during dipolarization. They also found that dipolarization occurs first between $-16<X<-11 R_{E}$ and then moves tailward at a rate of $35 \mathrm{~km} / \mathrm{s}$. According to Baumjohann et al. (1999), the tailward propagating dipolarization front typically reaches the vicinity of the near-Earth neutral line about $45 \mathrm{~min}$ after the onset of the substorm expansion phase. Because magnetic reconnection cannot operate in a dipolar magnetic field geometry, they concluded this marks the start of the substorm recovery phase.

\section{Study objectives}

Over the period from 1995 to 2000 , the Wind spacecraft spent over $500 \mathrm{~h}$ in the region of the Earth's magnetotail with Geocentric Solar Magnetospheric (GSM) coordinates of $-18<X<-6 R_{E}$ and $-16<Y<16 R_{E}$. Figure 1 illustrates the Wind trajectories in the $X-Y$ GSM plane for $X<0 R_{E}$ and $-6 R_{E}<Z<6 R_{E}$ from 1995 to 2000. Throughout this paper, GSM coordinates will be used to describe the positions of the Wind and GOES spacecraft, and for the magnetic field and plasma parameters measured in space. The passes made by Wind through the magnetotail from 1995 to 2000 are unique in that they consisted largely of west to east traversals of the near-tail beyond geosynchronous orbit at low latitudes. These passes are well-suited to studying the east-west extent of the substorm current wedge and the associated bursty bulk flows in the plasma sheet (e.g. Slavin et al., 1997).

The first objective of our study of the Wind magnetotail passes was to measure the statistical east-west extent of the 
substorm current wedge by examining the locations of magnetic field dipolarization events using data from the Wind Magnetic Field Investigation (MFI) (Lepping et al., 1995). The second objective was to determine how the properties of the magnetic field dipolarization events vary as a function of local time and distance to the neutral sheet. The third objective was to compare the properties of isolated dipolarization events to the properties of dipolarizations that occurred as part of a multiple dipolarization series. The characteristics of the dipolarization events that we examined included the change in the inclination of the magnetic field to the equatorial plane and the change in the total magnetic field. We also used the Wind 3-DP plasma measurements (Lin et al., 1995) to investigate the relationship between high-speed earthward flow bursts and magnetic field dipolarization events. The fourth objective of our study was to examine the timing between local observations of dipolarization events by Wind and substorm onset signatures. To accomplish this goal, we examined the intensity of auroral kilometric radiation (AKR) observed by the Wind WAVES instrument (Bougeret et al., 1995), and looked for dipolarizations in the GOES geosynchronous spacecraft magnetic field data. Magnetic field data from the CANOPUS network of ground magnetometers were also used when available.

\section{Event selection}

In the first round of dipolarization event selection, entire Wind passes through the magnetotail were scanned for dipolarization event candidates with increases in the $B_{z}$ component of the magnetic field and increases in the inclination angle of the magnetic field with respect to the $X-Y$ plane. No limits on the $Y$ position of the spacecraft were imposed during the initial selection, so that we could examine the full azimuthal extent of the substorm current wedge; however, only portions of the Wind orbit with $X<-6.0 R_{E}$ were considered. The candidate events found by visually searching for increases in $B_{z}$ and the magnetic field inclination were then screened to eliminate events where the change in magnetic field inclination was less than $15^{\circ}$. This selection condition was used to study dipolarizations in the AMPTE/CCE data set (Lopez et al., 1988). We used additional selection conditions based upon the results of a superposed epoch study of dipolarizations observed by Geotail (Baumjohann et al., 1999). Based upon the average magnetic field inclinations found by Baumjohann et al. (1999) for dipolarizations at distances between $-11<X<-16 R_{E}$ and $-16<X<-21 R_{E}$, we required that the maximum inclination angle had to be greater than $20^{\circ}$ and the ratio of the maximum to initial inclination had to be greater than 1.5 .

Because magnetic field dipolarization events and the flow bursts that accompany them are generally short-lived, sudden events, we also attempted to place conditions upon the rate of change of the magnetic field inclination during our dipolarization events. The durations of the earthward flow bursts and dipolarization events observed by Geotail at radial distances

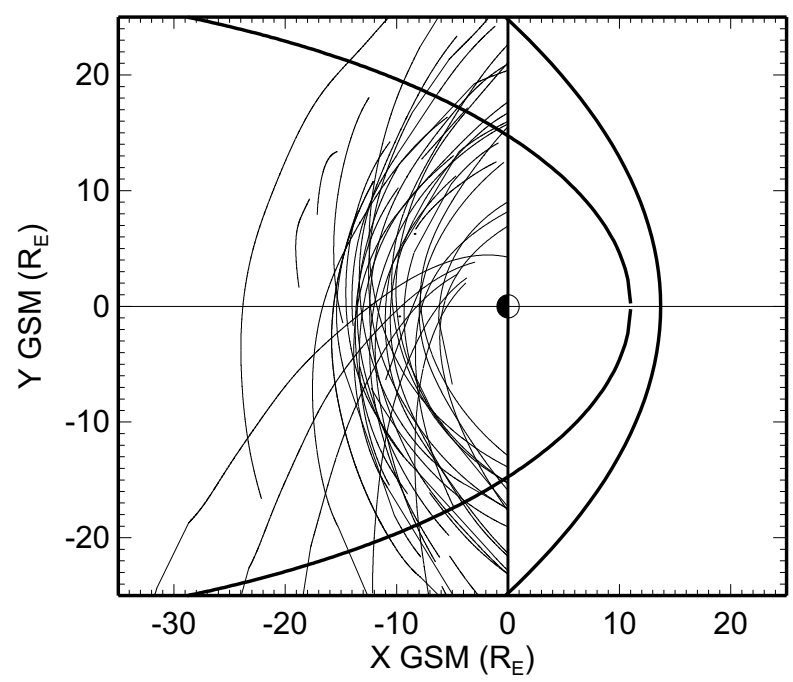

Fig. 1. Orbital trajectories of the Wind spacecraft in the $X-Y$ plane for 1995 to 2000 . The trajectory is shown only when $X<0 R_{E}$ and $-6 R_{E}<Z<6 R_{E}$.

Table 1. Dipolarization event selection conditions.

$\begin{array}{ll}\begin{array}{l}\text { Change in magnetic field inclination } \\ \text { (Based upon Lopez et al., 1988) }\end{array} & >15^{\circ} \\ \begin{array}{l}\text { Maximum inclination angle } \\ \text { (From the results of Baumjohann et al., 1999) }\end{array} & >20^{\circ} \\ \begin{array}{l}\text { Ratio of maximum inclination to initial } \\ \text { inclination (From the result of Baumjohann }\end{array} & >1.5 \\ \text { et al., 1999) } & \\ \begin{array}{l}\text { Rate of change of the magnetic field } \\ \text { inclination (this paper) }\end{array} & >1.5^{\circ} / \mathrm{min} \\ \end{array}$

of 10 to $13 R_{E}$ were on the order of $5-10 \mathrm{~min}$ (e.g. Sigsbee et al., 2002; Fairfield et al., 1998; Nagai et al., 2000). A minimum rate of change in the magnetic field inclination of $1.5^{\circ} / \mathrm{min}$ was initially chosen to aid in selecting Wind dipolarization events, since it would produce the required minimum $15^{\circ}$ change in inclination over a 10 -min time period. The condition on the minimum rate of change in the magnetic field turned out to be redundant, as nearly all of the events which satisfied the first three conditions also satisfied this one. The selection conditions applied to the Wind dipolarization event candidates in our study are summarized in Table 1. We identified 65 dipolarization events in the Wind magnetic field data set based upon these conditions. As we did not use any conditions based upon observations of global substorm activity to select the Wind dipolarization events, some of these dipolarization events may be pseudobreakups rather than full substorms. Wind also may not have been in the central plasma sheet during some of the events selected in this manner. To help clarify the difference between substorms and pseudobreakups, we categorized the dipolarization events as either isolated dipolarizations or part of a 
series of closely spaced dipolarizations. Isolated events were defined as dipolarizations observed at least $20 \mathrm{~min}$ before or after other dipolarizations. Dipolarization events were considered to be part of a series of multiple dipolarizations if they occurred less than $20 \mathrm{~min}$ apart.

\section{Substorm onset indicators}

One of the difficulties in studying substorms is choosing a measure of global substorm activity. Auroral kilometric radiation (AKR) is widely accepted as one indicator of auroral activity and substorms (e.g. Slavin et al., 2002). We decided to examine AKR observations from the Wind WAVES instrument during the Wind dipolarization events, since this is the one substorm indicator that was guaranteed to be available during all of the dipolarization events. AKR emissions cover a broad frequency range, so it is useful to examine a few select frequency bands or to integrate the power observed over the AKR frequency range when studying substorms. Because the frequencies at which AKR emissions first intensify were found to be highly variable during our dipolarization events, we chose to integrate AKR power over a broad frequency range.

Previous studies have integrated power in the AKR frequency range to construct a new index for geomagnetic activity. Murata et al. (1997) integrated Geotail Plasma Wave Instrument data from $50 \mathrm{kHz}$ to $800 \mathrm{kHz}$ to create an AKR index. The integrated AKR power index was normalized to a radial distance of $25 R_{E}$ from Earth. Murata et al. (1997) found that their AKR index was proportional to $K_{p}$ for $K_{p}<4$, and that there was a linear relation between the AKR index and $D_{s t}$ for $50<D_{s t}<0 \mathrm{nT}$. Kurth et al. (1998), used integrated plasma wave data from Polar to create an AKR index. Their AKR index was normalized to a radial distance of $9 R_{E}$, which is the apogee of the Polar spacecraft. Kurth et al. (1998) found a reasonable correlation between the AKR index and CANOPUS CU-CL index. They concluded that the integrated AKR power is a good proxy for the AE index. To construct an AKR index using Wind, we integrated Wind WAVES RAD1 data between $50 \mathrm{kHz}$ to $800 \mathrm{kHz}$. We normalized our AKR index to a radial distance of $14 R_{E}$, the average radial distance of the Wind dipolarization events.

Coverage by high-latitude ground magnetometer stations and geosynchronous spacecraft was limited during most of the Wind dipolarization events, due to local time constraints on the relevance of these observations to substorms. However, magnetic field data from the GOES 8 and 9 geosynchronous satellites in the night side magnetosphere were available during some of the Wind dipolarization events. In the case studies presented in this paper, data from the GOES geosynchronous satellites were used to examine the timing between the observations of dipolarizations and flow bursts at Wind with the start of the dipolarization at geosynchronous orbit. The properties of the dipolarizations, such as the $B_{z}$ increase and the increase in magnetic field inclination, observed by Wind and by GOES 8 or 9 , were also compared. Data from the CANOPUS ground magnetometer stations in Canada were also available during the case studies. The CANOPUS ground magnetometer data were used to provide an indication of closure of the substorm current wedge through the ionosphere.

\section{Case studies}

In this section, we will examine Wind data from four different magnetotail passes when magnetic field data from the GOES 8 and 9 geosynchronous spacecraft and CANOPUS ground magnetometer stations were available. These case studies illustrate examples of isolated dipolarizations and multiple dipolarization series events using the criteria described in Sect. 3. Comparison of the Wind magnetic field and plasma observations during these events with observations from GOES and the CANOPUS ground stations will allow us to explore the relation between the magnetic field dipolarizations in the mid-tail and at geosynchronous orbit with the development of the substorm current wedge.

\subsection{January 1996, 02:30-04:30 UT}

Figure 2 shows AKR observed by Wind WAVES, the $X$ (northward) component of the magnetic field measured at Fort Churchill in the CANOPUS ground magnetometer network, the GOES 8 magnetic field, the Wind magnetic field data, and Wind plasma data from 02:30 to 04:30 UT on 13 January 1996. Strong AKR emissions were observed by Wind throughout the time period shown in Fig. 2. At 02:51:00 UT, Wind observed a sudden intensification of the AKR emissions and the frequency range of the emissions extended to lower frequencies. The AKR data imply that intense auroral activity was already in progress more than $10 \mathrm{~min}$ before activity was observed by GOES 8 , and $40 \mathrm{~min}$ before the dipolarization was observed by Wind. Starting at 02:00 UT, the magnetic field inclination angle at GOES 8 steadily decreased from an initial value of $55^{\circ}$ to a value of $30^{\circ}$ at 03:00 UT. The decrease of the magnetic field inclination observed by GOES 8 indicates stretching of the near magnetotail during the growth phase (Nagai, 1982). Shortly after 03:00 UT, the $B_{z}$ component of the magnetic field and inclination angle at GOES 8 began to increase. GOES 8 was located near 22:02 LT at $(-5.3,3.6,-1.7) R_{E}$ when the dipolarization was observed at 03:02:25 UT. The dipolarization at GOES 8 is marked by a dotted line in Fig. 2 . The magnetic field inclination at GOES 8 reached a peak value of approximately $70^{\circ}$ at 03:43:30 UT. The GOES 9 spacecraft was located on the dusk flank near 18:00 LT at $(0.13,6.56,0.90) R_{E}$, where it observed low-frequency fluctuations of the magnetic field, but did not see a clear dipolarization signature.

An isolated dipolarization event was observed by the Wind spacecraft starting at 03:30:43 UT and is marked by the solid black line in Fig. 2. Prior to the start of the magnetic 
field dipolarization, Wind was located near 21:42 LT at $(-10.4,7.2,0.8) R_{E}$, in a quiet, tail-like region of the magnetic field, as indicated by the large, steady value of $B_{x}$ and the small value of $B_{z}$. These magnetic field signatures are similar to the growth phase signatures observed by GOES 8 prior to the start of the dipolarization at geosynchronous orbit. The magnetic field inclination to the equatorial plane at Wind was less than $5^{\circ}$ before the start of the magnetic field dipolarization. At the start of the magnetic field dipolarization, Wind observed a decrease in $B_{x}$ and the total magnetic field, while $B_{z}$ began to increase. At 03:50:52 UT the magnetic field inclination to the equatorial plane at Wind reached a maximum value close to $30^{\circ}$. Large amplitude fluctuations of the magnetic field were also observed after the start of the magnetic field dipolarization, similar to fluctuations of the magnetic field observed during dipolarizations by Geotail (Sigsbee et al., 2002; Nagai et al., 2000). Although Wind was located close to the equatorial plane during this event, the 3-DP plasma data indicate that the spacecraft was situated outside of a thinned plasma sheet before the dipolarization. A sudden increase in the plasma beta was recorded just after 03:40 UT, indicating that the plasma sheet had finally expanded over the spacecraft. The increase in the plasma beta was due to a slight decrease in the magnetic pressure and a sudden increase in the ion pressure by about a factor of 100. The total pressure at Wind's location in the magnetotail was dominated by the contribution of the magnetic pressure, so that the total pressure decreased only slightly during this dipolarization. The maximum earthward flow of $315 \mathrm{~km} / \mathrm{s}$ was observed by Wind at 03:45:34 UT, shortly after the plasma sheet expanded over the spacecraft, and near the time of the maximum magnetic field inclination to the equatorial plane. Although the earthward flows had a component parallel to the magnetic field, the component of the flows perpendicular to the magnetic field dominated during the largest earthward flow bursts.

The observations from Wind, at geosynchronous orbit, and on the ground, on 13 January 1996, show that even when an isolated dipolarization is observed in the near magnetotail, the timing of the local magnetotail reconfiguration relative to other onset signatures can be complicated. This could be due to the difference in the locations of the Wind and GOES spacecraft, and the manner in which the dipolarization spreads in local time and distance down the magnetotail. Comparison of the Wind and GOES 8 dipolarizations appear to indicate that the activity started close to midnight and then spread in local time. Similar conclusions were reached in the studies performed by Lopez et al. (1988) and Nagai (1991). However, it is not entirely clear how this expansion progressed. According to Nagai (1991), if the substorm onset occurs at midnight, a geosynchronous spacecraft near 22:00 MLT should see the dipolarization start less than $5 \mathrm{~min}$ after the onset. The dipolarization reached GOES 8 about $20 \mathrm{~min}$ after the AKR intensification and about $30 \mathrm{~min}$ before the dipolarization was observed by Wind. GOES 8 was located $20 \mathrm{~min}$ in local time closer to midnight than Wind, which could account for the observation of the dipolarization

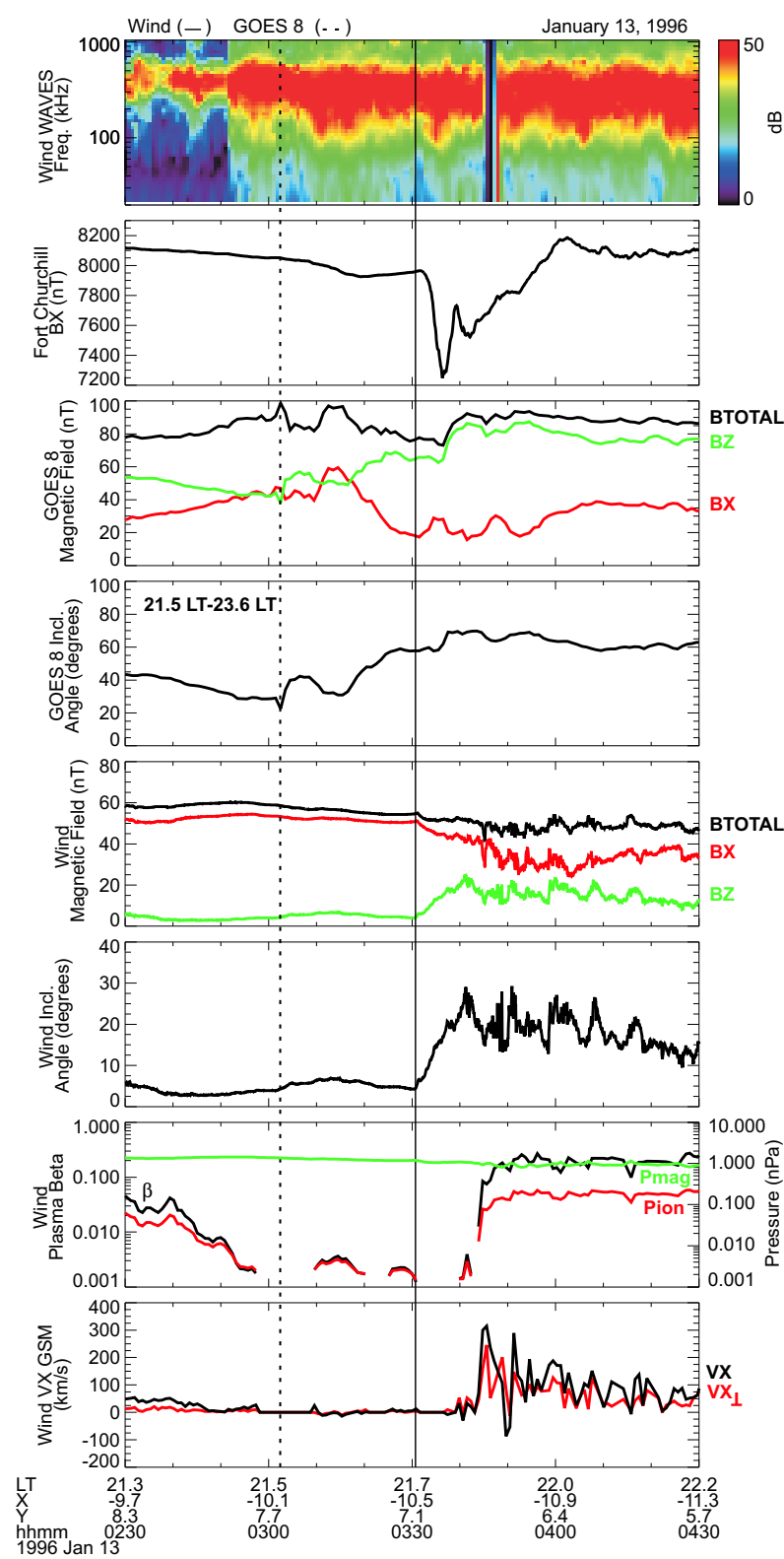

Fig. 2. Wind, CANOPUS and GOES 8 data from 02:30 to 04:30 UT on 13 January 1996. From top to bottom, Fig. 2 shows the Wind WAVES RAD1 receiver data, the $X$ (northward) component of the magnetic field measured at Fort Churchill, the total magnetic field (black) measured by GOES 8 , the $B_{x}$ (red) and $B_{z}$ (green) components of the magnetic field measured by GOES 8 , the magnetic field inclination at GOES 8 , the total magnetic field (black) measured by Wind, the $B_{x}$ (red) and $B_{z}$ (green) components of the magnetic field measured by Wind, the magnetic field inclination at Wind, the Wind plasma beta (black), the Wind ion (red) and magnetic (green) pressures, and the $X$ component of the flow velocity at Wind. The component of $V_{x}$ perpendicular to the magnetic field is shown in red and the total $V_{x}$ is shown in black. An isolated dipolarization event was observed by the Wind spacecraft starting at 03:30:43 and is marked by the solid black line. The start of the dipolarization observed by GOES 8 is marked by a dotted line. GOES 8 was located between $21.5 \mathrm{LT}$ to $23.6 \mathrm{LT}$ for the time period shown in the figure. 


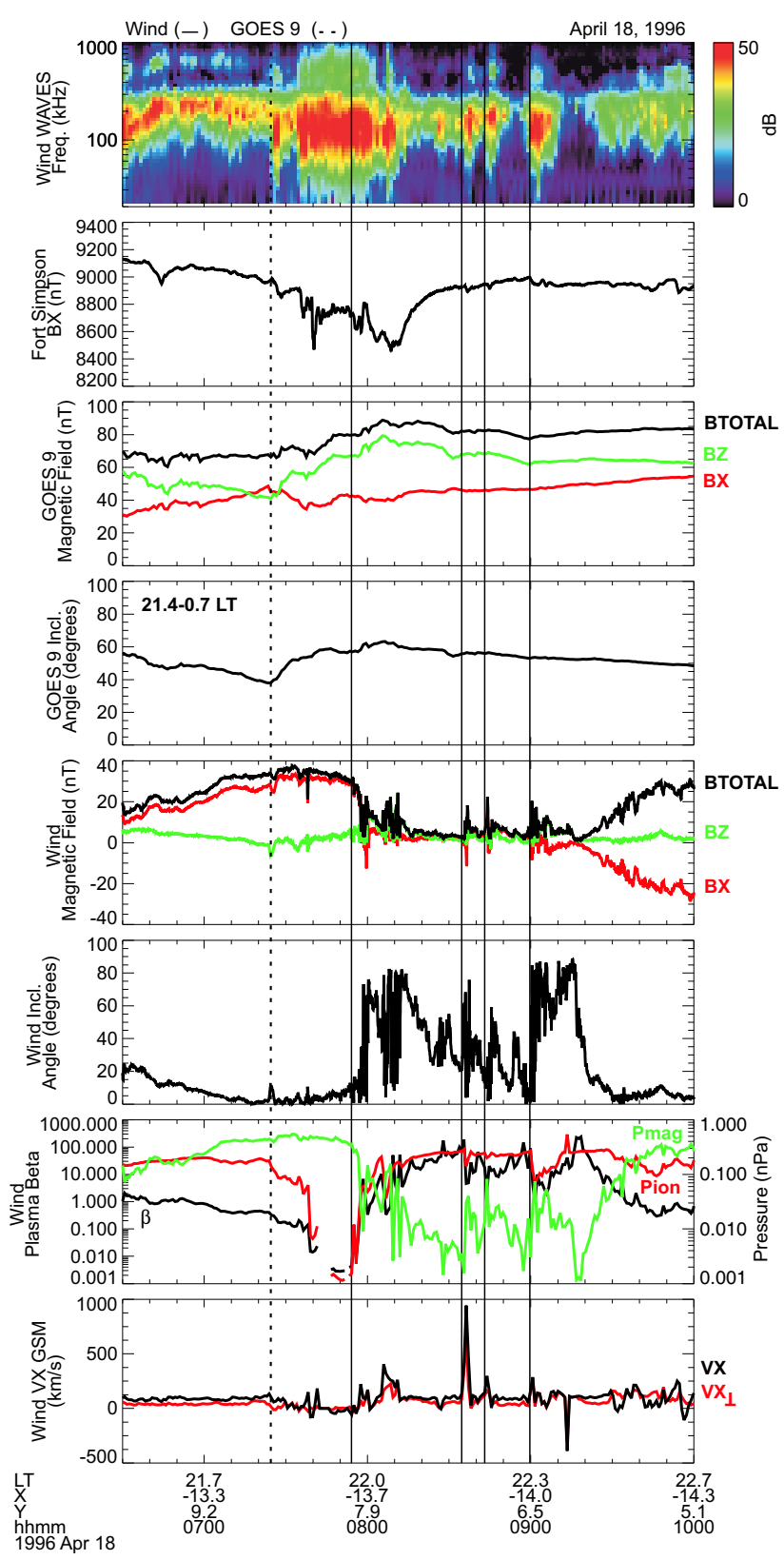

Fig. 3. Wind, CANOPUS and GOES 9 data from 06:30 to 10:00 UT on 18 April 1996. Figure 3 is the same format as Fig. 2, except that the $X$ component of the magnetic field from Fort Simpson is shown in the second panel and data from the GOES 9 geosynchronous satellite are shown. GOES 9 was located between 21.4 LT to 0.7 LT for the time period shown in the figure.

at GOES 8 before dipolarization started at Wind. However, this is not consistent with the Nagai (1991) study, which showed that it only takes about 30 min for a dipolarization at geosynchronous orbit to expand duskward from midnight to $18: 00$ MLT and about $15 \mathrm{~min}$ to expand dawnward to 04:00 MLT. Although the propagation and expansion of the dipolarization may change with radial distance, the difference between Wind and GOES 8 in local time seems to be too small to account for the 30-min delay in the start of dipolarization at Wind. It is interesting to note that the magnetic field inclination reached its peak values at both Wind and GOES 8 around 03:40-03:50 UT, even though the dipolarization at GOES 8 started 30 min earlier than the dipolarization at Wind. A negative bay developed at Fort Churchill in the CANOPUS ground magnetometer network a few minutes after the dipolarization occurred at Wind. There was a slight intensification in emissions at the lower end of the AKR frequency range associated with the dipolarization at Wind. These signatures indicate that the dipolarization at Wind represented a local intensification in the current system, rather than a new substorm onset.

\subsection{April 1996, 06:30-10:00 UT}

Figure 3 shows AKR observed by Wind WAVES, the $X$ (northward) component of the magnetic field measured at Fort Simpson in the CANOPUS ground magnetometer network, the magnetic field measured at GOES 9, the magnetic field measured by Wind, and Wind plasma data for the dipolarization events that occurred between 06:30 and 10:00 UT on 18 April 1996. This was a very active day, with a total of six dipolarizations observed by Wind from 05:00 to 11:00 UT which met the selection criteria in Table 1. Some of the dipolarizations observed by Wind on 18 April 1996 were separated by at least $20 \mathrm{~min}$ from preceding or succeeding dipolarizations and were classified as isolated events (dipolarizations at 05:24 UT, 07:54 UT, and 10:31 UT), while others were classified as part of a multiple dipolarization series (dipolarizations at 08:34 UT, 08:43 UT, and 08:59 UT). During the large flow bursts associated with the 07:54 UT and 08:34 UT dipolarizations, the component of $V_{x}$ perpendicular to the magnetic field (red trace in the last panel of Fig. 3) was quite large, although there was also a parallel component to these flow bursts. The earthward flow velocities were quite small $(<300 \mathrm{~km} / \mathrm{s})$ during the last three dipolarization events, even though plasma beta values greater than 1 indicated that Wind was located in the plasma sheet. However, the inclination angles still increased by more than a factor of 2 during these dipolarizations, and the maximum inclination angles reached were between $50^{\circ}$ and $90^{\circ}$. A possible explanation is that Wind was not located in the center of the flow channel, but was close enough to observe the magnetic field perturbations associated with the earthward transport of magnetic flux by the flows. This may be the most likely explanation since other studies (e.g. Angelopoulos et al., 1996, 1997a,b; Nakamura et al., 2004) have argued that flow bursts tend to occur in very localized regions of the magnetotail.

The time period from 07:00 to 10:00 UT on 18 April 1996 has also been examined in detail by Slavin et al. (1997), who compared the timing of the dipolarizations observed by Wind, Geotail, and GOES 9. The Wind WAVES instrument observed an intensification in AKR emissions from 07:33 UT to 08:10 UT. A negative bay was observed at Fort Simpson in the CANOPUS ground network simultaneously with the 
AKR intensification. GOES 9 was located near 22:16 LT at $(-5.9,2.7,0.8) R_{E}$ when it observed a magnetic field dipolarization at 07:24:30 UT, just before the start of the AKR intensification. The magnetic field inclination at GOES 9 increased from an initial value of $37.9^{\circ}$ to a maximum value of $63.3^{\circ}$ at 08:06:30 UT. At 07:54:07 UT, Wind was located near 21:55 LT at $(-13.7,7.7,1.2) R_{E}$ when an isolated dipolarization was observed. A few minutes earlier at 07:52 UT, a series of dipolarizations and earthward flow bursts were observed by Geotail near midnight and $X=-12.5 R_{E}$ (Slavin et al., 1997). The complicated timing between the dipolarizations observed by Wind, Geotail, and GOES 9 was interpreted by Slavin et al. as evidence for spatially localized, but temporally overlapping flow bursts in the near magnetotail. They suggested that the combined effects of the magnetic flux transported by these overlapping flow bursts caused the dipolarization observed by GOES 9 at geosynchronous orbit.

Although the data presented by Slavin et al. (1997) showed that this substorm was a complex event with multiple active regions in the near magnetotail, the properties of the 07:54:07 UT dipolarization observed by Wind were similar to the other isolated dipolarizations studied. Before the start of this dipolarization, Wind was located in a region of taillike magnetic field, as evidenced by the large $B_{x}$ component and the small $B_{y}$ and $B_{z}$ components of the magnetic field. The magnetic field inclination at Wind was only $3.6^{\circ}$ before the dipolarization started. A decrease in the Wind plasma beta before the start of this dipolarization indicated thinning of the plasma sheet. The decrease in the plasma beta during the growth phase was caused mainly by a gradual increase in the magnetic pressure from about $0.1 \mathrm{nPa}$ at $06: 30 \mathrm{UT}$ to about $0.5 \mathrm{nPa}$, just before the start of the dipolarization. A sudden decrease in the ion pressure from about $0.1 \mathrm{nPa}$ at 07:40 UT to about $0.001 \mathrm{nPa}$ at 07:50 UT also contributed to the decrease in the plasma beta just before the start of the dipolarization. At the start of the dipolarization, fluctuations of the magnetic field were observed, along with a decrease in $B_{x}$ and the total magnetic field, and an increase in $B_{z}$ and the magnetic field inclination. The sudden increase in the plasma beta at the start of the dipolarization, indicating expansion of the plasma sheet back over the spacecraft, resulted from a decrease in the magnetic pressure and sudden increase in the ion pressure. At 08:08:01 UT, the magnetic field inclination angle at Wind reached a maximum value of $82.5^{\circ}$. Earthward flows greater than $400 \mathrm{~km} / \mathrm{s}$ were observed by Wind at 08:06 UT, just before the maximum magnetic field inclination was reached.

Although the dipolarization started about 30 min earlier at GOES 9 than at Wind, the maximum inclination angle was reached at GOES 9 only about $1.5 \mathrm{~min}$ before the maximum inclination angle was reached at Wind. In both this dipolarization and the 13 January 1996 case study, the maximum inclination angle was reached at geosynchronous orbit only 1-2 min after the maximum earthward flow was observed by Wind, even though the magnetic field inclination at geosynchronous orbit began to increase much earlier than it did at Wind. The delay between the start of dipolarization at GOES and Wind during these events could be explained by the gradual formation of a region of high pressure and dipolarized magnetic field near geosynchronous orbit due to the effects of overlapping flow bursts in the magnetotail on the inner magnetosphere.

The 07:54 UT dipolarization observed by Wind was followed by a series of three smaller, closely spaced dipolarizations at 08:34 UT, 08:43 UT, and 08:59 UT. These three dipolarizations were interpreted as occurring during the substorm recovery phase by Slavin et al. (1997). In our study of dipolarizations, we labeled these three dipolarizations as part of a multiple dipolarization series, since they were spaced less than $20 \mathrm{~min}$ apart. These dipolarizations were not observed by the GOES 8 or GOES 9 spacecraft; however, they were associated with brief intensifications in AKR emissions and fluctuations in the magnetic field at Wind. These three dipolarizations were different from the isolated dipolarizations on 18 April 1996, as they did not show a typical growth phase period characterized by a thinned plasma sheet before the dipolarization. Wind remained in the plasma sheet for the entire time period between 08:30 and 09:30 UT, as indicated by the plasma beta. Between 08:30 and 09:30 UT, the ion pressure and total pressure remained nearly constant around $0.2 \mathrm{nPa}$. However, slight decreases in the ion pressure and total pressure occurred during the 08:59 UT dipolarization. Fluctuations in the plasma beta from 08:30 to 09:30 UT were caused by fluctuations of the magnetic pressure between values of 0.001 to $0.1 \mathrm{nPa}$. $B_{x}$ and the total magnetic field were small $(\sim$ a few nT) before the start of the 08:34 UT, 08:43 UT, and 08:59 UT dipolarizations. The change in magnetic field inclination was due mainly to the increase in $B_{z}$, and not due to a decrease in $B_{x}$ or the total magnetic field. This is fairly typical behavior for the dipolarizations that occurred as part of a multiple dipolarization series in our study, which will also be described during the discussion of the 26 July 1997 dipolarization events in Sect. 5.4, and in the results of the superposed epoch study described in Sect. 6.3.

\subsection{May 1996, 03:30-05:30 UT}

Another example of an isolated dipolarization event at Wind occurred on 10 May 1996 at 04:25:04 UT. Wind was located near 22:03 LT at $(-12.1,6.5,2.0) R_{E}$ during this dipolarization. Figure 4 shows AKR observed by Wind WAVES, the $X$ (northward) component of the magnetic field measured at Fort Churchill in the CANOPUS ground magnetometer network, the GOES 8 magnetic field data, the Wind magnetic field data, and the plasma data from Wind for this dipolarization event. The GOES 8 geosynchronous satellite was located near $22: 47 \mathrm{LT}$ at $(-6.1,1.5,2.0) R_{E}$ when it observed a magnetic field dipolarization. The dipolarization observed by GOES 8 occurred at 04:02:30 UT, more than $20 \mathrm{~min}$ before the dipolarization was observed by Wind. The dipolarization observed by GOES 8 at 04:02:30 UT featured a very steep increase in $B_{z}$ and the magnetic field inclination compared to the other examples of dipolarizations at geosynchronous orbit shown here. Only a small perturbation in $B_{x}$ 


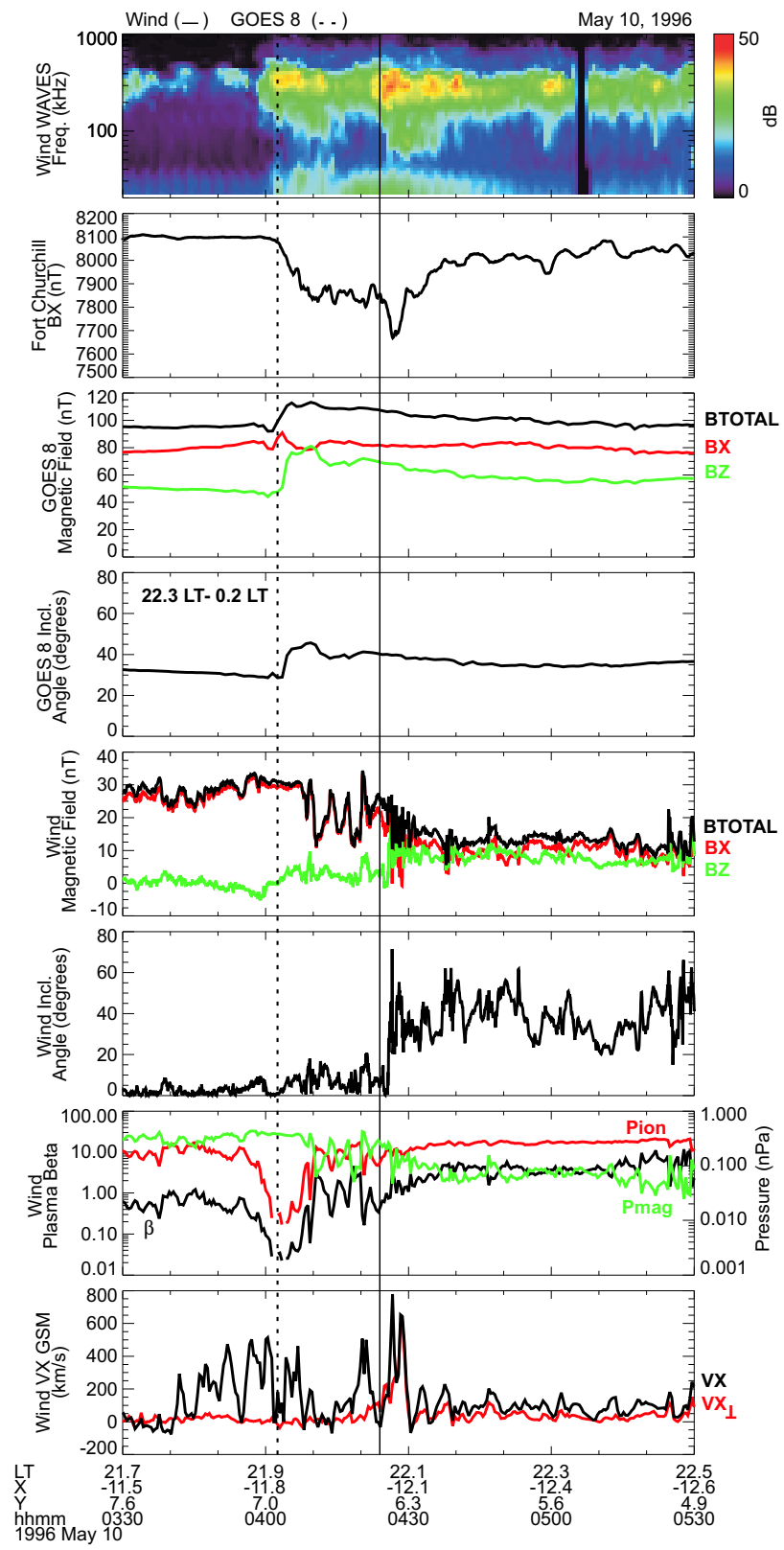

Fig. 4. Wind, CANOPUS and GOES 8 data from 03:30 to 05:30 UT on 10 May 1996. Figure 4 is the same format as Fig. 2. GOES 8 was located between $22.3 \mathrm{LT}$ to $0.2 \mathrm{LT}$ for the time period shown in the figure.

was observed by GOES 8 , so the increase in the magnetic field inclination was due mainly to the increase in $B_{z}$. The total magnetic field at GOES 8 also increased during this dipolarization. Increases in the magnetic field strength have also been reported in detailed studies of dipolarizations at geosynchronous orbit (e.g. Nagai, 1982). The magnetic field inclination at GOES 8 started at $28.6^{\circ}$ and then quickly increased to a maximum value of $45.7^{\circ}$ at 04:09:30 UT. After the maximum inclination was reached, the magnetic field inclination gradually decreased until about 05:00 UT. The observation of the dipolarization by GOES 8 was well correlated with an increase in the intensity of AKR emissions just before 04:00 UT, and with the development of a negative bay at Fort Churchill. Another AKR intensification occurred at 04:20 UT, just before the start of the dipolarization at Wind.

Bursts of earthward flow greater than $200 \mathrm{~km} / \mathrm{s}$ were observed by Wind from 03:30 UT to 04:10 UT. The flows observed from $03: 30$ to $04: 10$ UT were parallel to the magnetic field. These flows could represent the parallel flow in the plasma sheet boundary layer at the lobe-plasma sheet interface. The survey of high-speed bulk flows and field-aligned beams conducted by Raj et al. (2002) showed that bulk flows are generally perpendicular to the magnetic field close to the neutral sheet but can have a large field-aligned component away from the neutral sheet. After a short period when $V_{x}$ decreased dramatically, more earthward flow bursts were observed starting around 04:20 UT. The maximum earthward flow observed during this event was $776 \mathrm{~km} / \mathrm{s}$ at 04:26:38 UT, just after the start of the Wind magnetic field dipolarization at 04:25:04 UT. As the dipolarization progressed, the contribution of flows perpendicular to the magnetic field increased.

This dipolarization featured a decrease in $B_{x}$ and $B_{T O T A L}$, and a very steep increase in $B_{z}$ and the magnetic field inclination. The magnetic field inclination at Wind increased from an initial value of $0.2^{\circ}$ to a maximum value of $71.4^{\circ}$ in $1.5 \mathrm{~min}$. This implies that the magnetic field inclination changed at a rate of $47.5 \% \mathrm{~min}$. The maximum inclination was observed almost simultaneously with the maximum earthward flow during this dipolarization. The magnetic field fluctuated rapidly, and the maximum inclination of $71.4^{\circ}$ was reached only briefly. However, the average magnetic field inclination for the period from 04:25:04 UT to 05:10:00 UT was $38^{\circ}$, a significant increase over the initial value of $0.2^{\circ}$. Before the 10 May 1996 dipolarization, the magnetic pressure and total pressure remained fairly constant. The decrease in the plasma beta around 04:00 UT was due to a sudden drop in the ion pressure before dipolarization, similar to the decrease in the ion pressure before the 13 January 1996 dipolarization. During the 10 May 1996 dipolarization, the ion pressure increased, and the total pressure and magnetic pressure gradually decreased.

In most of the isolated dipolarizations, including the 10 May 1996 dipolarization, the total magnetic field observed by Wind decreased during the process of dipolarization. It is interesting to note that during the 10 May 1996, 04:02:30 UT dipolarization at GOES 8, and the 18 April 1996, 07:23:30 UT dipolarization at GOES 9, the total magnetic field at geosynchronous orbit increased during the dipolarization. Nagai (1982) attributed increases in the total magnetic field at geosynchronous orbit after dipolarization to the enhancement of the $H$ component of the magnetic field (parallel to the Earth's dipole axis and positive northward). At Wind's radial distance, the $B_{x}$ component of the magnetic field often dominates before an isolated dipolarization, and can be nearly a factor of 10 larger than $B_{z}$ when the magnetotail is highly stretched. During the 10 May 1996, event, the three components of the magnetic field measured by GOES 8 at geosynchronous orbit were within a factor of 2 of one 
another before the dipolarization. If the magnetotail is not highly stretched at geosynchronous orbit, one might expect to see a pile-up of magnetic flux during dipolarization, which would account for the increase in $B_{z}$, but no major decrease in $B_{x}$. This would also result in an increase in the total magnetic field at geosynchronous orbit.

\subsection{July 1997, 03:15-08:30 UT}

The period from 03:30 UT to 08:30 UT on 26 July 1997 is an example of a period with many pseudobreakups and dipolarizations, but nothing that looks like a classical, well-defined substorm (Fillingim et al., 2000, 2001). The activity during this time period was interpreted as consisting of a series of quiet-time pseudobreakups by Fillingim et al. (2000, 2001), due to the lack of significant poleward auroral expansion. However, the phenomena that occurred in the magnetotail were similar to those that occured during substorms. Wind observed ten dipolarizations that met the selection criteria in Table 1 on 26 July 1997 from 03:30 to 08:30 UT. The initial inclination angles were greater than $20^{\circ}$ in eight of the dipolarizations observed by Wind, and maximum inclination angles greater than $70^{\circ}$ were observed during five of the dipolarizations on 26 July 1997.

AKR observed by Wind WAVES, the $X$ (northward) component of the magnetic field measured at Fort Churchill, the magnetic field data from GOES 8, and the Wind magnetic field and plasma data for 26 July 1997, 03:30 to 07:30 UT are shown in Fig. 5. The dipolarizations observed by Wind were spaced quite close together (less than $20 \mathrm{~min}$ apart), so this time period includes many examples of dipolarizations labeled as part of a multiple dipolarization series in our study. The characteristics of many of the dipolarizations observed by Wind during this time period are considerably different from the properties of the isolated (spaced more than 20 min apart) dipolarization events. The definitions of isolated events and multiple dipolarization series events used in our study were chosen specifically to separate the type of dipolarization observed on 26 July 1997 and similar periods from the other events, and not to identify individual substorms. Some of the events labeled as isolated dipolarizations may in fact represent intensifications of an existing substorm, rather than the start of a new substorm.

Most of the isolated dipolarizations observed by Wind featured an increase in $B_{z}$, decreases in $B_{x}$ and $B_{T O T A L}$, and an increase in the magnetic field inclination. The decrease in $B_{T O T A L}$ appeared to be caused by the decrease in $B_{x}$ during most isolated events, which often began with a period of quiet, tail-like magnetic field just before the magnetic field dipolarization. The magnetic field often increased gradually as the density decreased just before an isolated event, indicating a thinning of the plasma sheet. Decreases in the plasma beta before the isolated events also indicated plasma sheet thinning, which is a well-known characteristic of the substorm growth phase in the magnetotail. Growth phase signatures were absent before many of the multiple dipolarization series events, such as those observed on 26 July 1997.

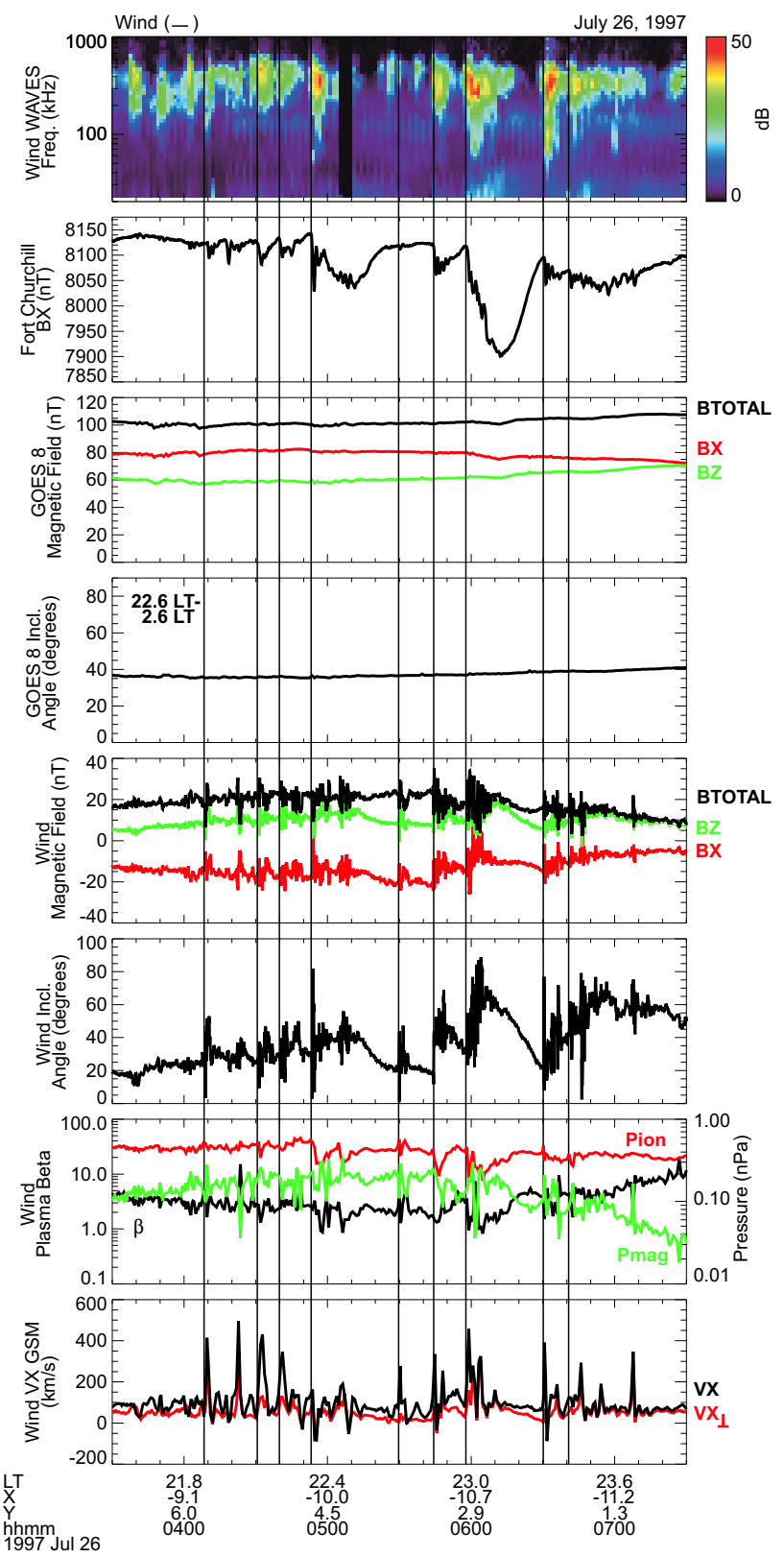

Fig. 5. Wind, CANOPUS and GOES 8 data from 03:30 to 07:30 UT on 26 July 1997. Figure 6 is the same format as Fig. 2. GOES 8 was located between 22.6 LT to 2.6 LT for the time period shown in the figure.

The plasma and magnetic field data for 26 July 1997, indicated that Wind was located in the plasma sheet for the entire interval when the dipolarizations were observed. The total pressure, magnetic pressure, and ion pressure remained fairly constant throughout the entire time interval shown, but small, transient fluctuations in these quantities occurred during some of the dipolarizations. During the other time periods considered, the ion pressure usually increased during dipolarization, as a result of plasma sheet expansion. However, on 26 July 1997, transient decreases in the ion pressure occurred during the dipolarizations at 04:53:07 UT, 05:44:19 UT, and 05:57:46 UT. 
It is interesting to note that from 04:00 UT to 05:20 UT, the average value of $B_{x}$ remained fairly steady, although large amplitude fluctuations of the magnetic field were observed during the dipolarization events. The $B_{z}$ component of the magnetic field started at about $5 \mathrm{nT}$ at 03:30 UT, and then began to gradually increase from 04:00 UT to 05:20 UT. Four perturbations in $B_{z}$ that resulted in brief increases in the inclination angle during this period were considered to be distinct dipolarization events (marked by vertical lines at 04:08 UT, 04:30 UT, 04:39 UT, and 04:53 UT in Fig. 5). Three of these dipolarizations were associated with large bursts of earthward flow. Although the magnetic field inclination and $B_{z}$ briefly increased and then decreased during each of these four dipolarizations, the inclination remained higher than its initial value after each dipolarization. It is not clear if these individual dipolarizations collectively were responsible for the gradual increase in $B_{z}$ observed from 04:00 UT to 05:20 UT, or if the $B_{z}$ increase was caused by something else and the dipolarizations were merely transient perturbations without any lasting effect.

After 05:20 UT, $B_{z}$ decreased suddenly, dropping to the same value as before the 04:08 to 04:53 UT dipolarizations. The magnitude of $B_{x}$ also increased slightly, but the plasma beta remained relatively steady and did not indicate significant thinning of the plasma sheet. Several minutes later, a series of three dipolarizations was observed by Wind (marked by vertical lines at 05:29 UT, 05:43 UT, and 05:57 UT in Fig. 5). Each of these dipolarizations increased in duration and intensity. The third dipolarization had the largest change in magnetic field inclination of the three, was associated with the largest flows, the largest AKR intensification, and had the longest duration. This series of dipolarizations was associated with a larger decrease in $B_{x}$ than the 04:00 to 05:20 UT dipolarizations, and each dipolarization appeared to contribute to the step-wise decrease in the magnitude of $B_{x}$ between 05:30 UT and 06:20 UT. Although the increase in the magnitude of $B_{x}$ between 05:20-05:29 UT indicated that there may have been some storage of energy in the magnetotail during this time, the decreases in $B_{x}$ and the behavior of the plasma beta after the 05:29 UT dipolarization are not consistent with the storage of additional energy in the magnetotail before the 05:43 UT and 05:57 UT dipolarizations. After the third dipolarization, $B_{z}$ began to decrease again until about 06:30 UT, when a final series of two dipolarizations was observed (marked by vertical lines at 06:30 UT and 06:40 UT). There was also a slight increase in the magnitude of $B_{x}$ before the 06:30 UT dipolarization, which could be interpreted as a short growth phase, but the plasma beta and the pressures do not indicate a thinning of the plasma sheet. The behavior of $B_{z}$ during these two dipolarizations was similar to the behavior of $B_{z}$ during the 04:00 to 05:20 UT dipolarizations. However, $B_{x}$ began to steadily decrease during the 06:30 UT dipolarizations.

Bursts of earthward flow were observed during most of the dipolarizations observed by Wind on 26 July 1997. Although the flow bursts each lasted only a few minutes, flows greater than $400 \mathrm{~km} / \mathrm{s}$ were observed during some of the dipolariza- tion events. These flow velocities are comparable to the flow velocities observed during isolated dipolarization events by Wind. In many of the flow bursts, the component of $V_{x}$ parallel to the magnetic field was quite large. Detailed analysis of the Wind particle distributions for 26 July 1997 showed that when $\left|B_{x}\right|$ was small, the flows were mainly perpendicular to the magnetic field, but when $\left|B_{x}\right|$ was larger, the flows were more field-aligned (Raj et al., 2002). Even when the flows observed on 26 July 1997 had a significant fieldaligned component, Raj et al. (2002) found that the flows still appeared to be convective in nature and were not consistent with field-aligned beams. All of the dipolarization events and flow bursts on 26 July 1997 were associated with brief intensifications of AKR and Pi2 pulsations at Fort Churchill. Some of the dipolarizations were also associated with the development of negative bays at Fort Churchill.

Although GOES 8 and GOES 9 were well-positioned to observe the geomagnetic activity on 26 July 1997, only very small perturbations of the magnetic field at geosynchronous orbit were observed. Neither GOES 8 nor GOES 9 observed dipolarization signatures during the activity observed by Wind on 26 July 1997. At geosynchronous orbit, Pi2 pulsations are observed less often during time periods with low $K_{p}$ index values than for periods with high $K_{p}$, due to the geometry of the inner magnetosphere (Singer et al., 1983). According to Singer et al. (1983), when $K_{p}$ is high, the inner boundary of the plasma sheet may be inside geosynchronous orbit, but when $K_{p}$ is low, the inner boundary of the plasma sheet and the Pi2 source region are located farther from Earth. The $K_{p}$ index was low on 26 July 1997, so the inner boundary of the plasma sheet may have been located too far tailward of geosynchronous orbit for dipolarizations to be observed by GOES 8 or 9 . Although no activity was observed at geosynchronous orbit, the dipolarizations observed by Wind on 26 July 1997 seem to be associated with most of the substorm expansion phase characteristics described in both the flow-braking/NENL and current disruption substorm models, so it is not clear why the magnetosphere does not follow the classical pattern of substorm development.

With the exceptions of the dipolarizations at 05:29 UT and 06:30 UT, the dipolarizations observed during the time period shown in Fig. 5 did not appear to be associated with typical growth phase signatures. A possible explanation is that solar wind conditions on 26 July 1997 caused the magnetosphere to enter into a pattern of directly driven activity where energy was continuously released in the magnetotail instead of stored in the tail lobes during a growth phase and then explosively released at the onset of the substorm expansion phase. However, the time period on 26 July 1997, shown in Fig. 5, does not fit the profile for a steady magnetospheric convection event. Solar wind data from Geotail at $(21.8,-13.9,-2.9) R_{E}$ and IMP-8 at $(27.2,-25.7,-2.4) R_{E}$ both indicated that $\left|B_{y}\right|$ was often larger than $B_{z}$ and that the IMF $B_{z}$ was small $(\sim 2 \mathrm{nT})$, and mainly northward, although it did fluctuate considerably during this time period. Steady magnetospheric convection 

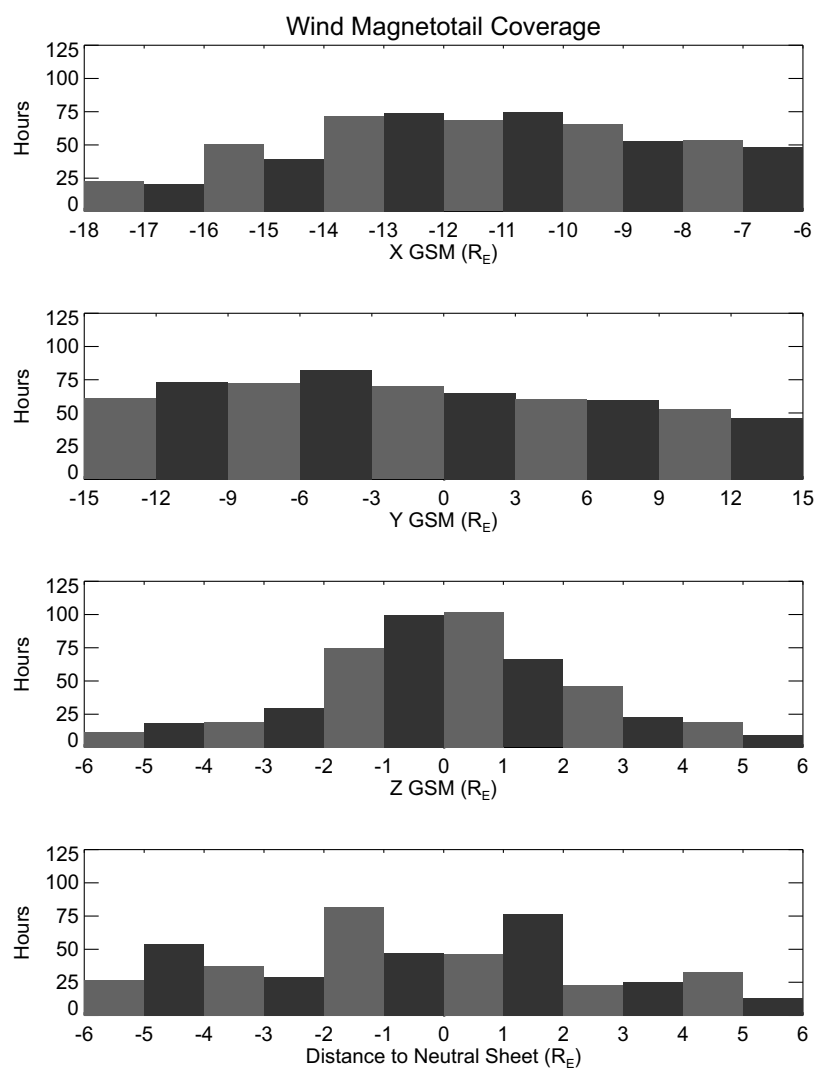

Fig. 6. The top 3 panels show histograms of the total amount of time in hours that the Wind spacecraft spent in different bins of the $X, Y$, and $Z$ GSM position of the spacecraft. The bottom panel shows the amount of time Wind spent at different distances from the neutral sheet as determined by Fairfield (1980).

events generally have several hours of southward IMF $B_{z}$ (e.g. Sergeev et al., 1996). When the propagation time delay is taken into account, it appears that brief, southward fluctuations in the IMF $B_{z}$ may have been associated with some of the dipolarization events observed by Wind on 26 July 1997. Due to the large distances of Geotail and IMP8 in the $Y$ direction and the rapid fluctuations in the IMF, it is difficult to determine with absolute certainty that these magnetic field fluctuations were associated with the dipolarizations observed by Wind. However, comparison with similar magnetotail activity observed by Geotail on 1 October 1996 when the IMF $B_{z}$ was small and fluctuating, suggests that the solar wind conditions were responsible for the continuous pattern of activity in the magnetotail on 26 July 1997 (Sigsbee et al., 2004).

\section{Statistical results}

\subsection{Spatial distribution of dipolarization events}

Figure 6 shows the total amount of time in hours that Wind spent in different regions of the magnetotail using GSM coordinates and the distance to the model neutral sheet de-
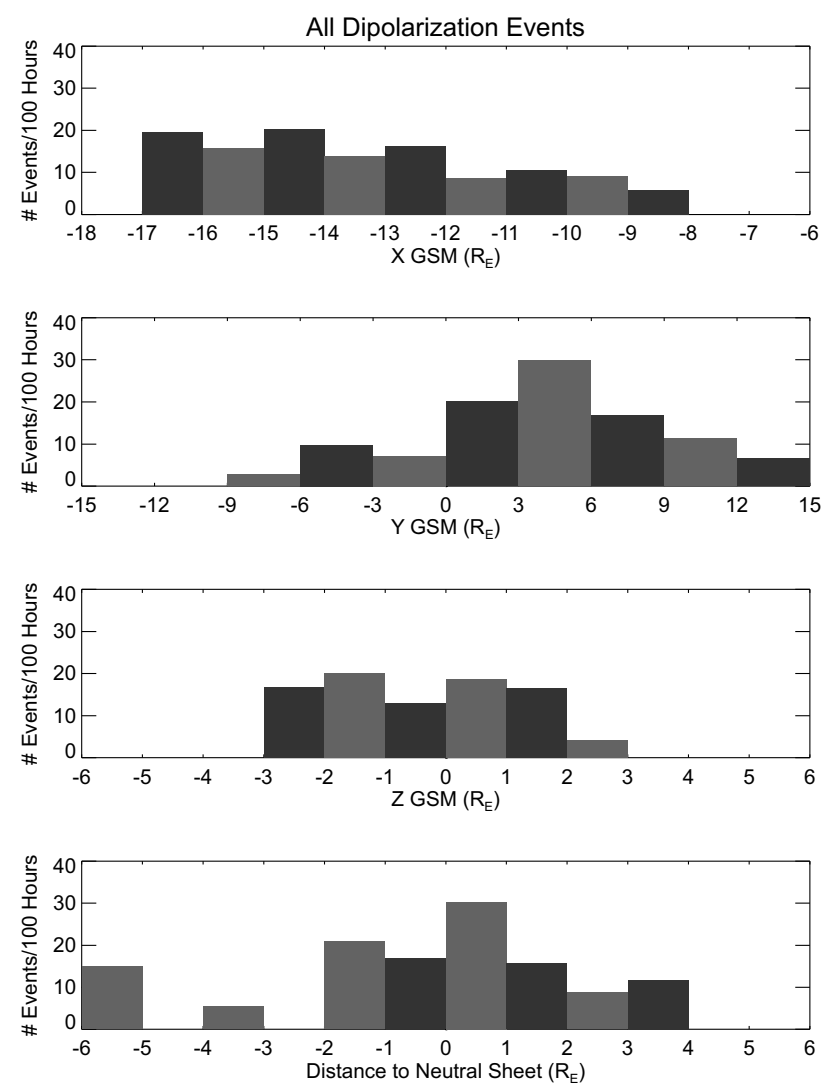

Fig. 7. Histograms of the number of dipolarization events per $100 \mathrm{~h}$ observed by Wind in the same position bins shown in Fig. 6.

termined by Fairfield (1980). The Wind spacecraft spent the most time in the magnetotail between $-6<X<-14 R_{E}$. The passes through the magnetotail were fairly evenly distributed across the tail in the $Y$ direction, but Wind spent slightly more time in the post-midnight region $(Y<0)$ than it did in the pre-midnight region. Wind was generally located between $-2<Z<3 R_{E}$, but there was a much broader distribution of positions relative to the Fairfield (1980) neutral sheet, as shown in Figure 6. Seasonal effects caused by the Earth's dipole tilt were taken into account when calculating the neutral sheet model and the position of Wind relative to the neutral sheet.

Figure 7 shows a histogram of the number of dipolarization events per $100 \mathrm{~h}$ observed by Wind in the position bins from Fig. 6. Dipolarization events were observed by Wind between $-8<X<-17 R_{E}$. More dipolarization events were observed further away from Earth, in agreement with results from AMPTE/CCE (Lopez et al., 1988). Although Wind spent more time in the post-midnight region of the magnetotail, the distribution of dipolarization events was peaked in the pre-midnight region $(Y>0)$, confirming the results of the Lopez et al. (1988) dipolarization study. The dipolarization events were all observed between $-3<Z<3 R_{E}$, and most occurred within a few $R_{E}$ of the Fairfield (1980) neutral sheet. 

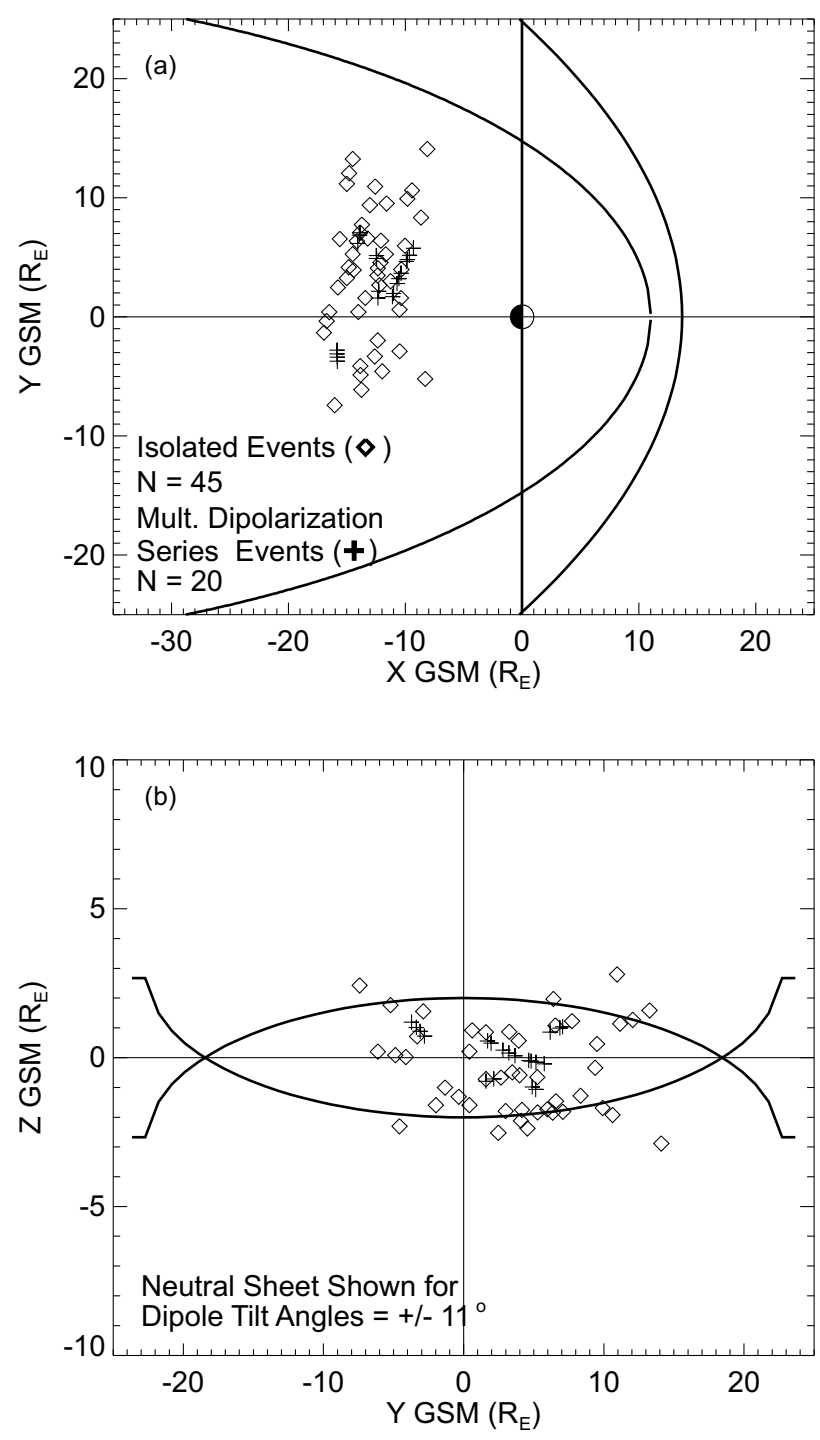

Fig. 8. The spatial distribution of the 45 isolated dipolarization events and 20 multiple dipolarization series events observed by Wind in (a) the $X-Y$ and (b) the $Y-Z$ GSM planes. Isolated dipolarization events were defined as dipolarization events occurring at least $20 \mathrm{~min}$ after the last dipolarization event and at least $20 \mathrm{~min}$ before the next dipolarization event. Dipolarization events that were spaced less than 20 min apart were considered to be part of a multiple dipolarization series. The two curves plotted in the $Y-Z$ plane represent the Fairfield (1980) neutral sheet for dipole tilt angles of $\pm 11^{\circ}$.

Figure 8 shows the spatial distribution of the 65 dipolarization events observed by Wind in the $X-Y$ and $Y-Z$ GSM planes. Figure 8a illustrates that most of the dipolarization events observed by Wind occurred in the premidnight region. The distribution of the dipolarization events in the $X-Y$ GSM plane is similar to the spatial distribution of high-speed $(>250 \mathrm{~km} / \mathrm{s})$ bulk flow events reported in a survey of 17 Wind perigee passes between 1995 and 1997 (Raj et al., 2002). As described in Sect. 3, isolated dipolarization events were defined as dipolarization events occurring at least $20 \mathrm{~min}$ after the last dipolarization event and at least $20 \mathrm{~min}$ before the next dipolarization event. Although this may not correspond to the usual definition of an isolated substorm, making this distinction seemed to be appropriate for the activity observed by Wind in the magnetotail. We found that the distribution of the events that occurred as part of a multiple dipolarization series was more localized in the premidnight region than the distribution of the isolated dipolarization events, with most multiple dipolarization series events occurring close to midnight. As many of the multiple dipolarization series events occurred during quiet times, this appears to be consistent with the findings of Lopez et al. (1988), that the center of the substorm current wedge shifts from the pre-midnight region towards the post-midnight region with increasing geomagnetic activity. It is also consistent with studies showing that pseudobreakups are more limited in spatial extent than substorms (Nakamura et al., 1994). In Fig. 8b, the two curves plotted in the $Y-Z$ plane represent the Fairfield (1980) neutral sheet for dipole tilt angles of $\pm 11^{\circ}$. As shown in Fig. 8b, most of the dipolarization events occurred close to the neutral sheet, in agreement with earlier studies (Lopez et al., 1988; Baumjohann et al., 1999).

\subsection{Observations of earthward flows}

We did not require the presence of high-speed earthward flows in the selection of dipolarization events, but Wind 3-DP plasma data (Lin et al., 1995) showed that $78 \%$ of the events had more than $5 \mathrm{~min}$ of earthward flow greater than $100 \mathrm{~km} / \mathrm{s}$. Maximum earthward flow velocities greater than $300 \mathrm{~km} / \mathrm{s}$ were observed in $57 \%$ of the events. When only isolated dipolarization events were considered, this percentage increased to $60 \%$. Although earthward flow was not a selection condition in our initial search for dipolarization events, it would appear that most dipolarization events are accompanied by earthward flow. This is in agreement with other studies of magnetic field dipolarization events, such as Baumjohann et al. (1999) and Shiokawa et al. (1998). Figure 9 shows when the maximum earthward flow velocity was observed relative to the start of the magnetic field dipolarization for the isolated events. As shown by Fig. 9, the maximum earthward flow velocity was observed within 10 to $12 \mathrm{~min}$ after the start of the dipolarization during most of the isolated events. The maximum earthward flow occurred before the start of the dipolarization in only one isolated event.

The second panel in Fig. 9 shows the number of events in 2 min bins of the difference between the time of the maximum inclination angle and the start time of the dipolarization for differences from -10 to $+30 \mathrm{~min}$. The second panel shows that the maximum magnetic field inclination is typically reached within $18 \mathrm{~min}$ after the start of dipolarization. The third panel in Fig. 9 shows the number of events in 2 min bins of the difference between the time of the maximum inclination angle and the time of the maximum earthward flow velocity $V_{x}$ for differences from -10 to $+30 \mathrm{~min}$. In two of the events, the maximum inclination angle was reached more than 10 min before the maximum $V_{x}$ was observed, so they 
fall outside the limits of the plot. The third panel shows peaks in the distribution between 0 and $4 \mathrm{~min}$, indicating that in most cases, the maximum inclination angle was reached almost simultaneously with the observation of the maximum earthward flow velocity or just a few minutes later. In $28 \%$ of the events shown in the third panel, the maximum inclination angle was reached before the maximum $V_{x}$ was observed.

A number of explanations exist for the cases where the maximum inclination was reached before the peak flow: 1) Wind was located on tail-like field lines and did not observe the flows until the plasma sheet expanded over the spacecraft; 2) the flows were weak ( $\sim 100 \mathrm{~km} / \mathrm{s})$ and fluctuating; 3) the magnetic field was fluctuating rapidly, so the maximum magnetic field inclination was not sustained for very long. Substorm observations by Geotail also featured variable flow directions and magnetic field fluctuations during the flows (Nagai et al., 2000). Explanations (2) and (3) probably can account for many of the cases in which the maximum inclination was reached before the peak flow. However, there is another possible explanation why large flows were not observed during some events. The presence of large fluxes of highenergy ions that exceed the operating limits of typical particle instruments has been observed in the magnetotail during substorms (see Fairfield et al., 1998; Nagai et al., 2000). The effects of particles penetrating the detector were taken into account when the Wind 3-DP data were analyzed, but it is possible that quantities such as the velocity and density were underestimated due to these particles. For more details on the plasma flows and particle distributions observed by Wind in the magnetotail, consult the comprehensive study conducted by Raj et al. (2002).

In $72 \%$ of the events shown in Fig. 9 the maximum inclination angle was reached after the maximum flow velocity was observed. Figure 9 supports the flow-braking picture described by Shiokawa et al. $(1997,1998)$ reasonably well, since in most cases the peak flow was observed before the dipolarization reached its maximum inclination angle. This is also consistent with observations from Geotail at $X$ GSM $>-20 R_{E}$, in which plasma flows were observed to start before the increase in $B_{z}$ associated with magnetotail dipolarization (Nagai et al., 2000). Figure 9 also shows that the dipolarization observed by a single spacecraft in the magnetotail is generally a rapid process, occurring on time scales less than the duration of the auroral disturbances associated with a typical substorm, which can last for more than $1 \mathrm{~h}$, as shown by global auroral images (e.g. Mende et al., 2003, and references therein).

\subsection{Superposed epoch study of dipolarizations and AKR}

To perform a superposed epoch study of the Wind magnetic field dipolarization events, we divided the magnetotail between $-18<X<-8 R_{E}$ into bins $3 R_{E}$ wide in $Y$. We assumed that the substorm expands symmetrically around midnight, and grouped events in the pre-midnight and postmidnight regions at the same $|Y|$ together. We started by examining 42 isolated dipolarization events ( $>20$ min apart)
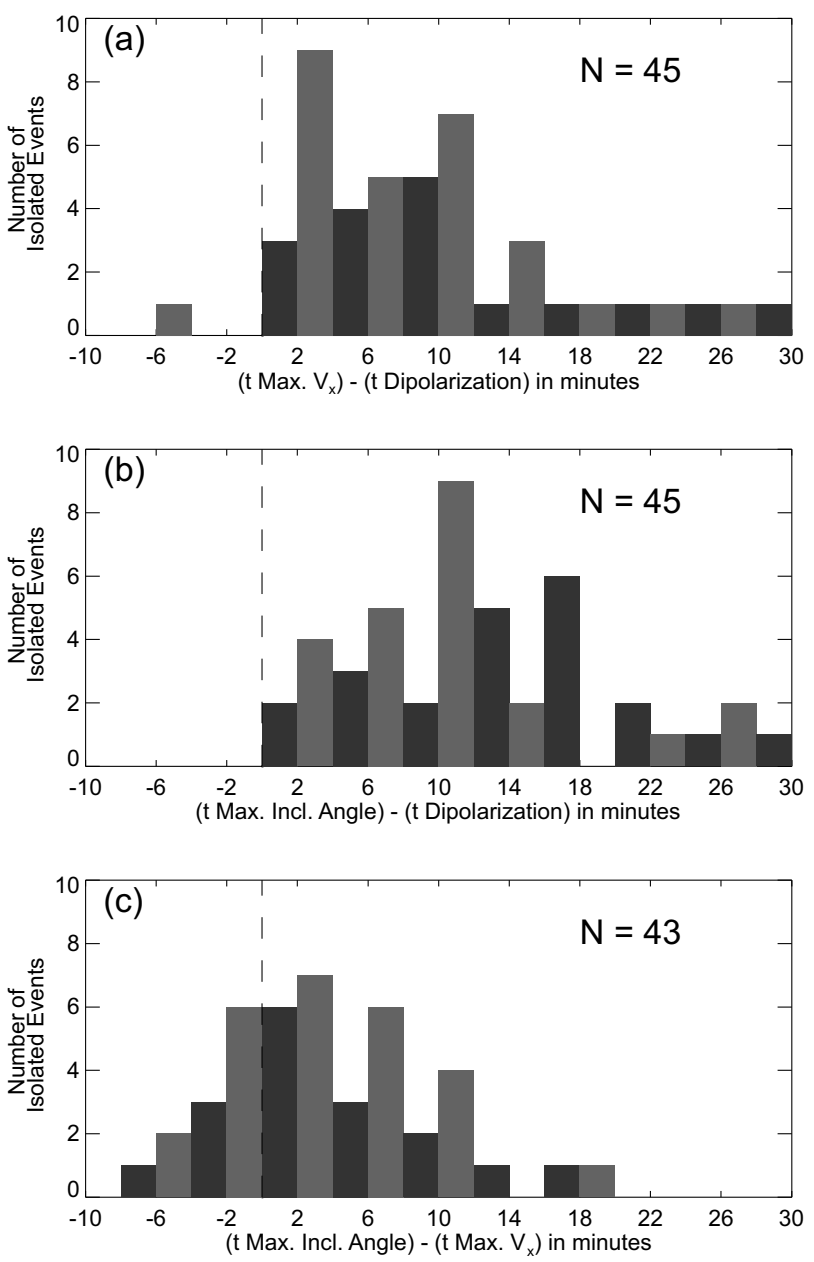

Fig. 9. (a) A histogram of the number of isolated events in $2 \mathrm{~min}$ bins of the difference between the time when the maximum earthward flow velocity was observed and the start of the magnetic field dipolarization. (b) The number of events in 2 min bins of the difference between the time when the maximum inclination angle was observed and the start time of the dipolarization. (c) The number of events in 2 min bins of the difference between the time when the maximum inclination angle was observed and the time when the maximum earthward flow velocity was observed.

between $0<|Y|<12 R_{E}$. We attempted to use the integrated AKR power index as an indicator of global substorm onset times for a superposed epoch study of the azimuthal expansion of the substorm current wedge, but the timing of the Wind dipolarization events relative to the AKR intensifications was not well-defined. We found that it was difficult to identify onset times correctly using the AKR index even for isolated dipolarization events because the highly variable frequency and bandwidth of AKR made it difficult to chose a suitable intensity threshold. We therefore chose the zeroepoch time to be the start of the $B_{z}$ increase, to examine how the AKR varied relative to the magnetic field dipolarization and to see how the properties of the dipolarization changed with the $Y$ position. Figure 10 shows the results of the superposed epoch study for the isolated events. We used $1 \mathrm{~min}$ 

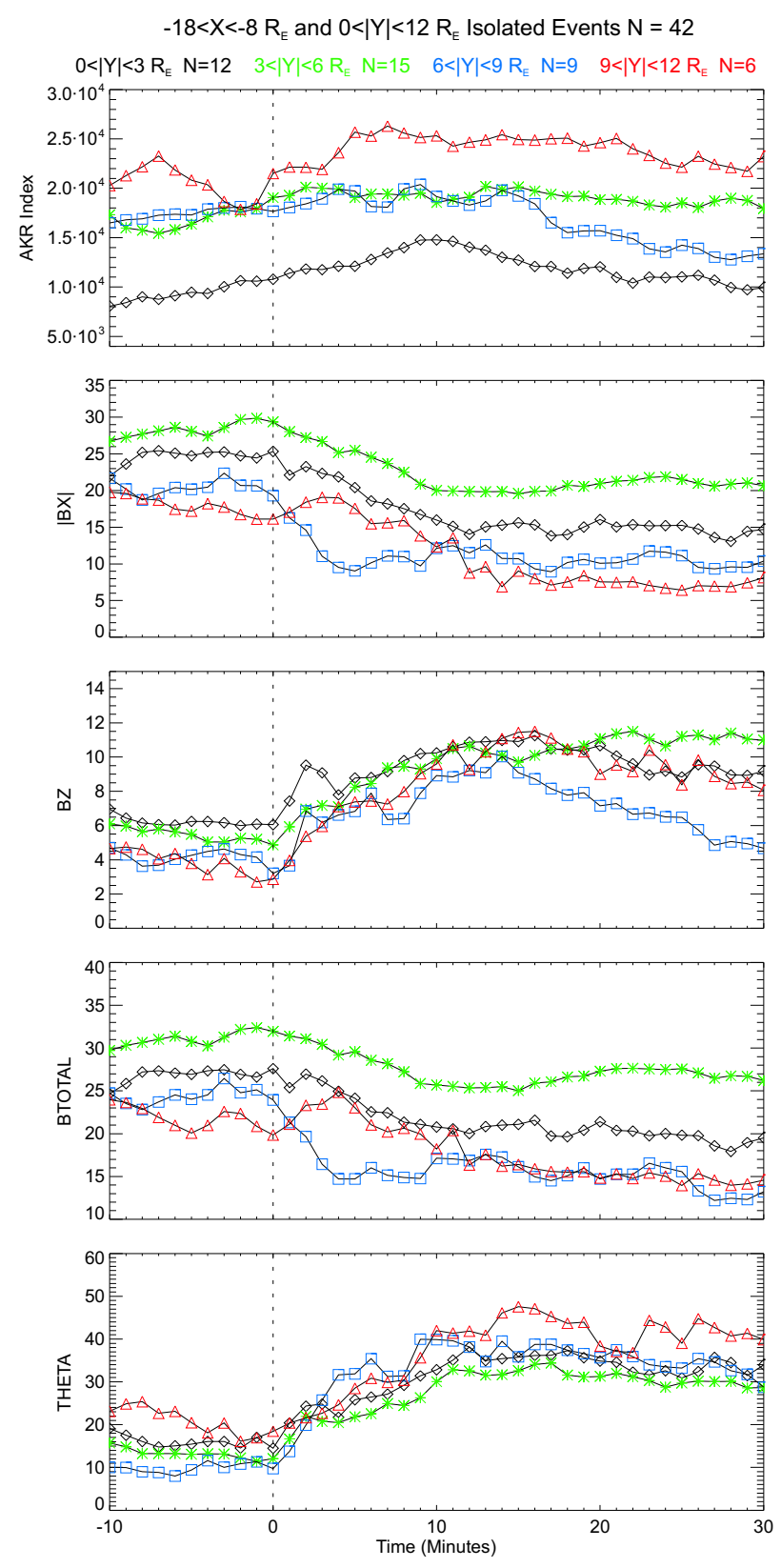

Fig. 10. Results of the superposed epoch study showing the dependence of dipolarization properties on the $Y$ GSM position for the isolated events. We used $1 \mathrm{~min}$ averages of the magnetic field in the regions $0<|Y|<3 R_{E}, 3<|Y|<6 R_{E}, 6<|Y|<9 R_{E}$, and $9<|Y|<12 R_{E}$. The absolute value of $B_{x}$ is plotted in Fig. 10 to prevent $B_{x}$ from averaging to zero. The AKR index was obtained by integrating the Wind WAVES data as described in Sect. 3.

averages of the magnetic field in the regions $0<|Y|<3 R_{E}$, $3<|Y|<6 R_{E}, 6<|Y|<9 R_{E}$, and $9<|Y|<12 R_{E}$. Only 3 events were observed from $12<|Y|<15 R_{E}$, so we did not include this region in the superposed epoch study. The absolute value of $B_{x}$ is plotted in Fig. 10 to prevent $B_{x}$ from averaging to zero, since some of the events started in the northern tail lobe (positive $B_{x}$ ) while others started in the southern tail lobe (negative $B_{x}$ ). The data are plotted starting $10 \mathrm{~min}$ be- fore the dipolarization and ending $30 \mathrm{~min}$ afterward.

The traces for the different $|Y|$ regions in Fig. 10 do not appear to be ordered in any particular sequence. This suggests that the properties of the isolated magnetic field dipolarizations are fairly uniform across the width of the magnetotail. The lack of systematic organization in the $|Y|$ direction also implies that the local regions of activity in the magnetotail could vary significantly for different events. During the 18 April 1996 and 26 July 1997 case study events, several dipolarizations were observed at nearly the same local time and radial distance, but the characteristics of the dipolarizations were highly variable. The lack of systematic organization in the $|Y|$ direction could also be an indication that the state of the magnetotail prior to dipolarization is more important than local time in determining how dipolarization proceeds in the mid-tail. However, it is still possible to identify some general characteristics of the isolated dipolarizations in this region of the magnetotail from Fig. 10. The isolated dipolarization events were characterized by an increase in $B_{z}$, an increase in the magnetic field inclination angle, and decreases in $\left|B_{x}\right|$ and $B_{T O T A L}$ starting approximately at the same time as the $B_{z}$ increase. The change in magnetic field inclination to the equatorial plane appeared to be closely correlated with the $B_{z}$ increase, while the decrease in the total magnetic field was more strongly correlated with the decrease in $\left|B_{x}\right|$. The decrease in $B_{T O T A L}$, shown in Fig. 10, agrees with the results from AMPTE/CCE, which showed that the total magnetic field decreases after most dipolarizations (Lopez et al., 1988).

As shown in Fig. 10, there does not appear to be any correlation between the AKR index and the increase in the magnetic field inclination during dipolarization for the isolated dipolarizations. It is possible that in some cases the AKR emissions did not reach Wind due to the shielding effects of the plasmasphere. However, studies of AKR propagation show that these emissions can be observed near the equatorial plane in the night-side magnetosphere, provided that the spacecraft is not close to the plasmapause and a broad frequency range is considered (Gurnett, 1974; Green et al., 1977; Gallagher et al., 1979). As the average radial distance of the Wind dipolarization events was $14 R_{E}$ and we used the frequency range $50 \mathrm{kHz}$ to $800 \mathrm{kHz}$, the influence of AKR propagation effects on our results was probably not significant.

To be certain that grouping events by $|Y|$ did not lead us to an erroneous conclusion about the dependence of the dipolarization properties on position in the $Y$ direction, we repeated the analysis using $3 R_{E}$ bins of $Y$ between $-9<Y<12 R_{E}$. The results of the superposed epoch analysis using bins of $Y$ were nearly identical to the results using $|Y|$. The traces for different $Y$ regions also did not appear to be ordered in any particular sequence, and there was not a clear correlation between the start of dipolarization and the behavior of the AKR index. This result was quite surprising, as studies of dipolarizations at geosynchronous orbit (e.g. Nagai, 1991) suggest that we should have found a clear time delay for dipolarizations observed far away from midnight. Nagai (1991) found 
that at geosynchronous orbit, dipolarization occurs first in a narrow local time sector center at 23:30 MLT, and that the region of dipolarization eventually expands longitudinally eastward and westward, reaching 03:00 MLT in $11 \mathrm{~min}$ and 20:00 MLT in $15 \mathrm{~min}$. This suggests that there may be some important differences in how magnetic field dipolarizations develop at geosynchronous orbit and in the region of the magnetotail between $-18<X<-8 R_{E}$ where the Wind dipolarization events were observed. However, multiplespacecraft data from Cluster in the post-midnight region of the magnetotail at GSM positions of $(-14.5,-11.6,4.2) R_{E}$ on 22 July 2001 and $(-18,-6.7,2.3) R_{E}$ on 12 August 2001 indicated that the dipolarization fronts during these events moved earthward and dawnward with speeds of $\sim 77 \mathrm{~km} / \mathrm{s}$ and $\sim 83 \mathrm{~km} / \mathrm{s}$, respectively (Nakamura et al., 2002a,b). The directions of propagation found during the Cluster studies are consistent with the expansion described for geosynchronous orbit by Nagai (1991). However, the separations between the Cluster spacecraft were only a few thousand $\mathrm{km}$, which is comparable to what is thought to be the typical size of the earthward flow bursts observed during substorms, but small compared to the entire scale of the magnetotail. With spacecraft separations of this size, it is difficult to be certain if these observations represented an expansion of the dipolarization front in local time, or just the propagation of a localized disturbance.

We also examined the dependence of the dipolarization properties on the distance to the neutral sheet using the Fairfield (1980) neutral sheet model. The results are shown in Fig. 11. We once again used $1 \mathrm{~min}$ averages of the magnetic field, only this time we grouped events together in the regions $-18<X<-8 R_{E}, 0<|Y|<12 R_{E}$. We assumed the properties of the dipolarizations would be the same above and below the neutral sheet, and organized the data into $1 R_{E}$ bins of the distance $\left|Z_{N S}\right|$ from Wind to the Fairfield (1980) neutral sheet model between $0<\left|Z_{N S}\right|<6 R_{E}$. The absolute value of $B_{x}$ was once again used to prevent $B_{x}$ from averaging to zero. As expected, the data show there is a strong dependence of the dipolarization properties on the distance to the neutral sheet. Smaller initial values of $\left|B_{x}\right|$ were observed closer to the neutral sheet, while the largest initial values of $\left|B_{x}\right|$ were observed in the $5<\left|Z_{N S}\right|<6 R_{E}$ bin. The change in $\left|B_{x}\right|$ after dipolarization also appeared to be the largest in the $5<\left|Z_{N S}\right|<6 R_{E}$ bin. The behavior of $B_{T}$ OTAL closely resembled the behavior of $\left|B_{x}\right|$ as a function of the distance to the neutral sheet. The values of $B_{z}$ were fairly similar in all position bins, but there appeared to be a slight trend towards a larger change in $B_{z}$ in the bins furthest away from the neutral sheet. A strong dependence of the inclination angle on the distance to the neutral sheet was observed. The greatest change in the magnetic field inclination was observed in the $0<\left|Z_{N S}\right|<1 R_{E}$ bin, while the smallest change in magnetic field inclination occurred in the $5<\left|Z_{N S}\right|<6 R_{E}$ bin. The substorm signatures and dipolarization characteristics observed at geosynchronous orbit have also been found to depend on the distance to the equatorial plane, as shown by variations in the radial component of the magnetic field
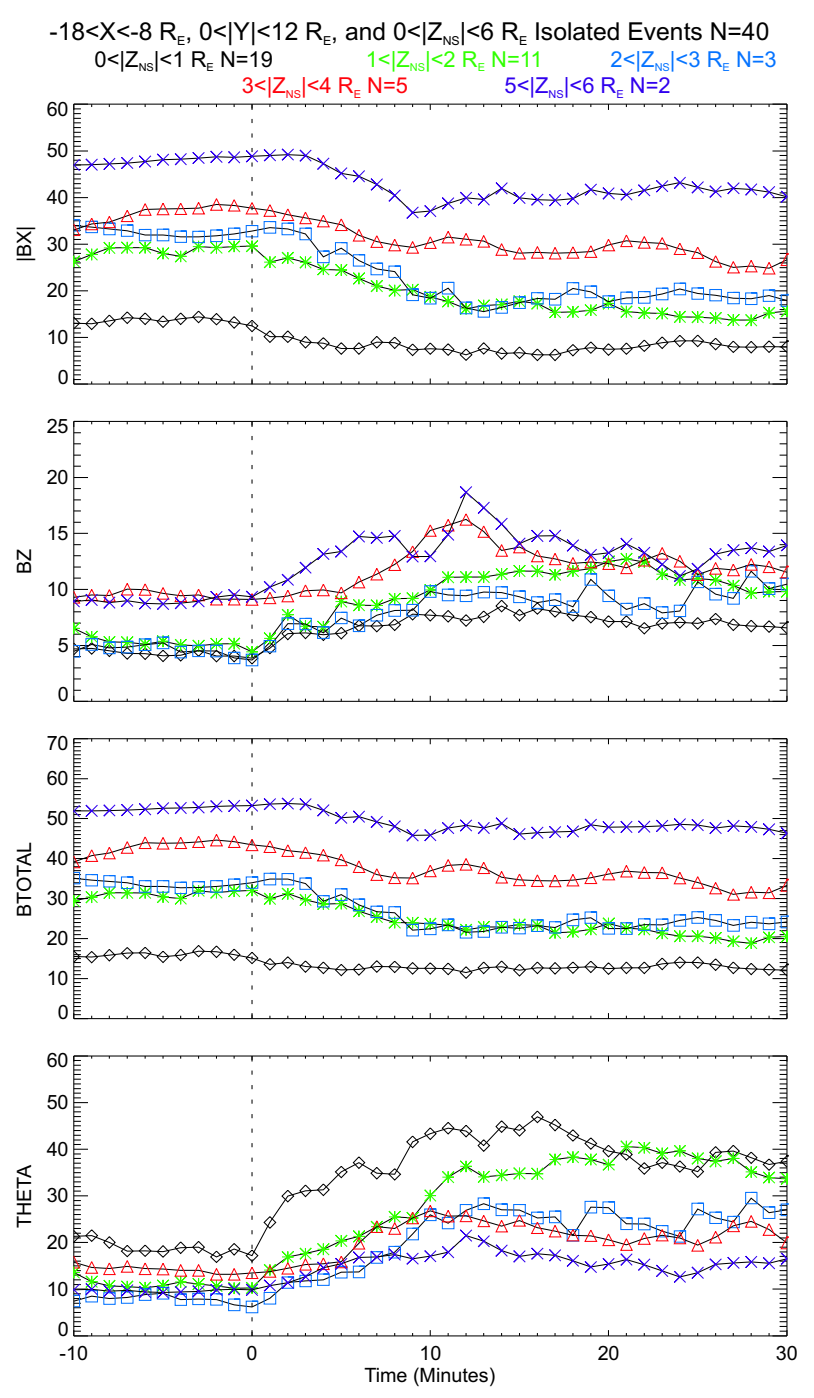

Fig. 11. Results of the superposed epoch study for the isolated events showing the dependence of dipolarization properties on the distance to the neutral sheet. We used 1 min averages of the magnetic field in the regions $-18<X<-8 R_{E}, 0<|Y|<12 R_{E}$. The absolute value of $B_{X}$ is plotted in Fig. 10 to prevent $B_{X}$ from averaging to zero.

measured by the ATS- 1 and ATS- 6 spacecraft (Kokubun and McPherron, 1981).

The results of superposed epoch analysis comparing the properties of isolated dipolarization events ( $>20 \mathrm{~min}$ apart) and events that were part of a multiple dipolarization series $(<20 \mathrm{~min}$ apart) are shown in Fig. 12. To compare the isolated dipolarization events and events that occurred as part of multiple dipolarization series, we once again grouped events in the pre-midnight and post-midnight regions at the same $|Y|$ together. We examined only events between $-16<X<-9 R_{E}$ and $0<|Y|<9 R_{E}$, since no multiple dipolarization series events were observed outside this region. We used $1 \mathrm{~min}$ averages of the magnetic field in Fig. 12. The zero epoch was defined as the start of the $B_{z}$ increase as before; however, the time span of the plots is 

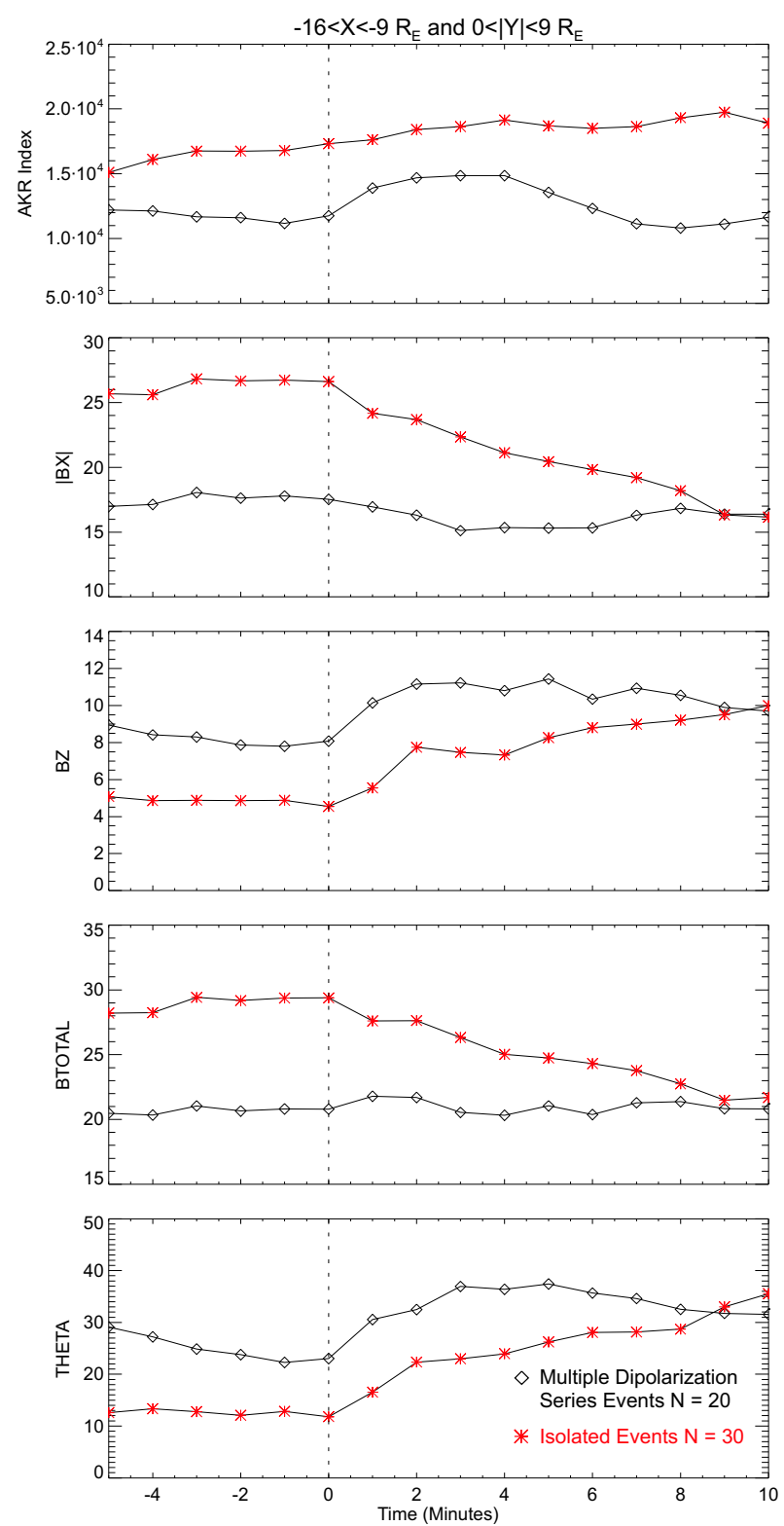

Fig. 12. Superposed epoch comparison of the magnetic field changes during the multiple dipolarization series events and isolated dipolarization events. We used $1 \mathrm{~min}$ averages of the magnetic field in the regions $0<|Y|<9 R_{E}$ and $-16<X<-9 R_{E}$. The absolute value of $B_{x}$ is plotted to prevent $B_{X}$ from averaging to zero.

from $5 \mathrm{~min}$ before the start of dipolarization to $10 \mathrm{~min}$ after the start of dipolarization. There were some key differences in the properties of the isolated dipolarizations and multiple dipolarization series events. We found that $\left|B_{x}\right|$ and $B_{T} O T A L$ generally decreased in the isolated cases, but they remained fairly steady in the events that were part of a multiple dipolarization series. The magnitude of $B_{x}$ and $B_{T O T A L}$ was also smaller in the multiple dipolarization series events. Although the total magnetic field decreased in most of the dipolarizations observed by AMPTE/CCE, it remained steady in $20 \%$ of the events (Lopez et al., 1988). It is possible that the AMPTE/CCE dipolarizations in which the total magnetic field did not show either a sustained increase or decrease after onset were small, pseudobreakup-type events similar to our multiple dipolarization series events.

The inclination angle and $B_{z}$ increased in both the isolated dipolarizations and multiple dipolarization series cases. However, in the multiple dipolarization series events, $B_{z}$ and the inclination angle peaked about $4 \mathrm{~min}$ after the start of the dipolarization and then began to decrease. Both $B_{z}$ and the inclination angle still appear to be increasing at $10 \mathrm{~min}$ after the dipolarization started in the isolated events. As shown in Fig. 12, the AKR intensity in the multiple dipolarization series cases increased at the start of dipolarization, reached a maximum at about 3 min after the start of dipolarization and then began to decrease. However, in the isolated cases, there was not a clear relationship between the AKR index and the dipolarization. A possible explanation is that when multiple, sequential dipolarizations occur at nearly the same local time in the mid-tail, each dipolarization in the series and the accompanying flow bursts result in only a transient, localized auroral intensification, while the isolated cases appear to be associated with activity on a more global scale.

Past studies have shown that pseudobreakups and substorms feature essentially the same physical phenomena, but on different temporal and spatial scales (Nakamura et al., 1994; Fillingim et al., 2000). In the Wind data set, the character of the multiple dipolarizations series events, or pseudobreakups, was fundamentally different from the isolated cases, which appear to be more consistent with substorm onsets or intensifications. The differences in the behavior of $\left|B_{x}\right|$ and $B_{\text {TOTAL }}$ between the isolated and multiple dipolarization series events suggests that the amount of energy stored in the magnetotail before dipolarization is quite different in these two types of events. The close spacing of the multiple dipolarization series events also suggest that there is limited time for a growth phase between these dipolarizations. The multiple dipolarization series events discussed in this paper are not to be confused with multiple onset substorms. During the substorms with multiple expansion phase onsets studied by Nagai et al. (1983), activity progressed in a step-wise fashion, and successive onsets appeared to occur in overlapping regions of local time. Nagai et al. (1983) also found that during substorms with multiple expansion phase onsets, each onset appeared to have its own growth period, in which the magnetic field became more tail-like at geosynchronous orbit. However, there did not appear to be a prolonged growth phase at Wind's location immediately before most of the multiple dipolarization series events on 26 July 1997 or before the dipolarizations observed during the substorm recovery phase on 18 April 1996.

The decision to divide the Wind dipolarization events into multiple dipolarization series events spaced less than $20 \mathrm{~min}$ apart and isolated events spaced more than 20 min apart was based solely on the characteristics of the dipolarizations observed by Wind. It is therefore quite interesting that the 20 min time scale chosen to divide our events into two categories is the same as the time scale determined by Bargatze 
et al. (1985) for direct coupling between the solar wind and magnetosphere. Using a linear prediction filter technique, Bargatze et al. (1985) examined the temporal relationship between the AL index and the solar wind input function $V B_{S}$ determined from IMP-8 data. They found two response pulses, peaked at time lags of 20 and $60 \mathrm{~min}$. The $20 \mathrm{~min}$ response was interpreted as magnetospheric activity that was directly driven by the solar wind, while the $60 \mathrm{~min}$ response was interpreted as magnetospheric activity that was driven by the release of energy previously stored in the magnetotail. Bargatze et al. (1985) found that the amplitude of the $60 \mathrm{~min}$ response pulse was larger for moderate activity levels, while the $20 \mathrm{~min}$ response pulse amplitude was larger for strong activity levels. Although Bargatze et al. (1985) concluded that directly driven activity occurs most often during periods of strong geomagnetic activity, it appears that the unusual pattern of magnetotail activity on 26 July 1997 may also be directly driven by the solar wind, even though it occurred during a geomagnetically quiet period. The lack of a welldefined growth phase before most of the dipolarizations, the occurrence frequency of the dipolarizations, and the fluctuations observed in the solar wind all support this interpretation for the 26 July 1997 activity. However, other multiple dipolarization series events included in our study may not represent this type of activity, even though the magnetic field behaved in a similar fashion during dipolarization. For example, the three closely spaced dipolarizations observed starting at 08:34 UT during the 18 April 1996 case study were associated with the recovery phase of a large substorm. These recovery phase dipolarizations could still be involved in the redistribution and dissipation of the energy previously stored in the magnetotail during the substorm growth phase.

\section{Discussion}

If the earthward flows associated with magnetic field dipolarizations are braked by pressure gradients in the magnetotail at the boundary between the tail-like and dipolar field regions, one would expect that very little earthward flow would be observed after the maximum magnetic field inclination to the equatorial plane was reached at Wind. Once the dipolarization front has propagated tailward past Wind's location, the braking point of the earthward flows should also be located tailward of Wind, so that no additional earthward flows would be observed. This was generally true for the Wind dipolarization events, but in two of the case studies presented in this paper the maximum $V_{x}$ occurred after the maximum magnetic field inclination angle was reached. The first case was the dipolarization observed at 03:30:43 UT on 13 January 1996. Before this dipolarization, Wind was located outside of a thinned plasma sheet, as indicated by the low plasma beta, and a magnetic field inclination of less than $5^{\circ}$. The earthward flows were only observed after the magnetic field dipolarization caused the plasma sheet to expand over the spacecraft. The second case where the maximum flow velocity was observed after the maximum inclination angle was reached occurred at 04:39:49 UT on 26 July 1997. The time period on 26 July 1997 shown in Fig. 5 was very unusual because Wind observed a series of many small dipolarizations, which were generally spaced less than 20 min apart, but no major substorm expansion occurred. Wind was located in the plasma sheet for the entire time period, shown in Fig. 5, and most of the dipolarizations on 26 July 1997 did not feature a distinct growth phase period before the start of the dipolarization. It is not clear why the timing of the maximum flow relative to the dipolarization was different during the 04:39 UT dipolarization. With these two exceptions, the Wind observations during the case study periods were generally consistent with a scenario in which the dipolarization results from a pile-up of magnetic flux carried earthward by high-speed flows in the plasma sheet.

Although the isolated dipolarizations observed at Wind typically featured decreases in $B_{x}$ and $B_{T O T A L}$, the dipolarization properties appear to be somewhat different at geosynchronous orbit. During some of the case studies presented here, the total magnetic field actually increased at geosynchronous orbit after the magnetic field dipolarization. A full discussion of the properties of dipolarizations at geosynchronous orbit is beyond the scope of this paper. However, the comparison between the Wind and GOES observations during the case studies has important implications for substorm physics. During the 18 April 1996 case study, only one dipolarization was seen during a given substorm at geosynchronous orbit by the GOES spacecraft, even when multiple dipolarizations and flow bursts were observed by Wind. It appears that the earthward flows observed at Wind's location in the near-tail can build-up a region of dipolarized magnetic field and high pressure that expands in local time and crosses GOES once at geosynchronous orbit. In other words, Wind sees multiple disturbances in the near magnetotail that are integrated by the inner magnetosphere to produce the signatures of the substorm current wedge at geosynchronous orbit and on the ground. Further evidence to support this idea is provided by the timing of the dipolarizations observed by Wind and GOES 8 on 10 May 1996. The maximum magnetic field inclination was reached nearly simultaneously at both spacecraft, even though the dipolarizations began at different times. This appears to suggest a gradual increase in pressure at geosynchronous orbit caused by activity at larger radial distances. The statistical results presented here and by Lopez et al. (1988) showing that a larger number of dipolarizations are observed with increasing radial distance provide even more support for this theory.

The behavior of the pressure in the magnetotail during the Wind case study events indicates that the variations in pressure associated with dipolarizations can be quite complicated. During the isolated dipolarizations on 13 January 1996, 10 May 1996, and 18 April 1996, it appeared that the magnetic pressure and total pressure decreased slightly during dipolarization, while the ion pressure increased. However, at geosynchronous orbit, the total magnetic field can increase during dipolarization, which implies a magnetic pressure increase in this region. During the multiple onset 


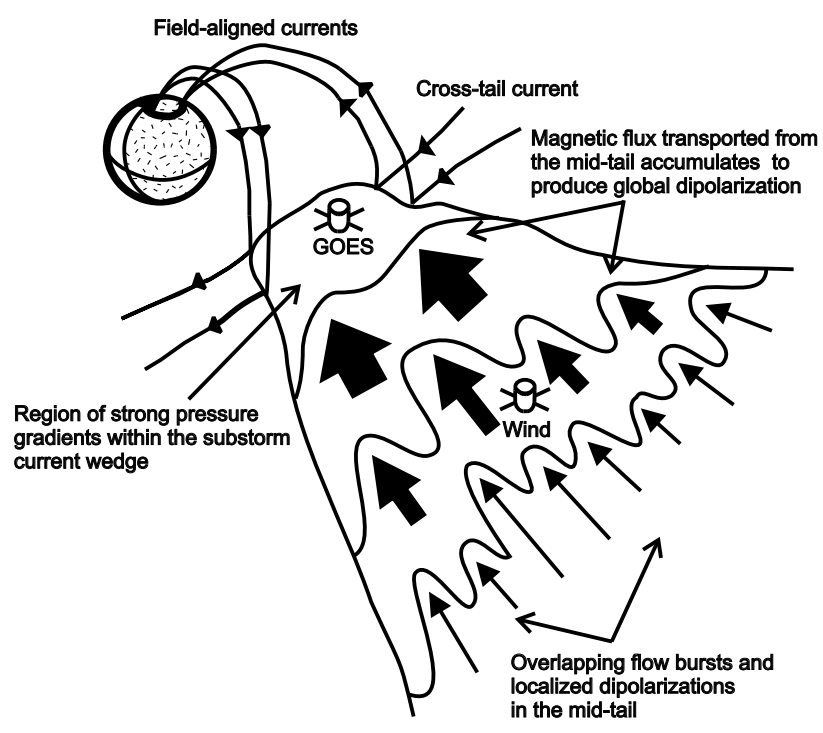

Fig. 13. Diagram illustrating the development of the substorm current wedge through overlapping flow bursts and localized dipolarizations. In this scenario, dipolarization of the entire magnetotail is a piecewise process resulting from the accumulation of magnetic flux transported by spatially and temporally overlapping flow bursts. The pressure gradients associated with these flow bursts are integrated by the inner magnetosphere to produce the substorm current wedge.

dipolarizations on 18 April 1996 and 26 July 1997, the total pressure and magnetic pressure remained nearly constant, but the ion pressure decreased during some events. A study of the evolution of the magnetotail pressure during substorms using data from Geotail indicated that a decrease in the total pressure begins near $(X, Y)=(-18,7) R_{E}$ just before substorm onset and then spreads to surrounding regions of the magnetotail (Miyashita et al., 1999). However, Miyashita et al. (1999) also found that a total pressure enhancement can occur in the region near $X \sim-10 R_{E}$ and $-5<Y<5 R_{E}$ during dipolarization. Pressure gradients in the magnetotail are important to substorms, but the evolution of the magnetotail pressure during substorms is difficult to characterize using single spacecraft measurements, as the observed behavior appears to depend strongly on the spacecraft location. External factors, such as the solar wind dynamic pressure, as well as internal magnetospheric processes, can also affect the magnetotail pressure (see Nakai and Kamide, 2004, and references therein).

The dipolarization of the magnetotail that occurs during substorms does not appear to be a single, sudden, global event as commonly believed. As evidenced by the duration of the AKR emissions observed by Wind, the auroral activity and current systems associated with substorms often persist for much longer time periods than the duration of the flows and isolated dipolarizations observed by a spacecraft in the near tail. Studies of earthward flow bursts have indicated that the flows may also be spatially limited, in addition to occurring on relatively short time scales compared to substorms.
Recent multiple-spacecraft observations from Cluster have provided direct estimates of the spatial scales of the flow channels and dipolarization front at distances greater than $-10 R_{E}$. Using data from the four Cluster spacecraft near $(-14.5,-11.6,4.2) R_{E}$ on 22 July 2001, Nakamura et al. (2002b) determined that the dipolarization front leading the flow burst had a nearly planar configuration, suggesting that the spatial scale of the flow burst should be much larger than the maximum separation of the spacecraft $(2000 \mathrm{~km})$. This is consistent with the typical spatial scales of 1 to $5 R_{E}$ for bursty bulk flows predicted by other studies (Angelopoulos et al., 1997b, 1994). Using multi-point observations of earthward flow bursts $\left(V_{\perp}>300 \mathrm{~km} / \mathrm{s}\right)$ from Cluster at distances $X<-15 R_{E}$ between July and October 2001, Nakamura et al. (2004) directly determined that the full width of the flow channel is 2 to $3 R_{E}$ in the dawn-dusk direction and 1.5 to $2 R_{E}$ in the north-south direction. In the 13 January 1996 event, the 18 April 1996 event, and the 10 May 1996 event, the dipolarizations at geosynchronous orbit all began before dipolarizations were observed by Wind. If flow bursts in the magnetotail are indeed localized to the narrow channels determined in the Cluster studies, it is possible that the flow bursts and localized dipolarizations in the mid-tail associated with the initial onsets during these events did not occur close enough to Wind to be observed.

The lack of clear correlations between the start of dipolarization in the magnetotail and AKR intensifications during the isolated dipolarizations also suggest that global dipolarization during substorms is a slow, piecewise process that instantaneously involves only localized portions of the magnetotail. In the 13 January 1996 event, the 18 April 1996 event, and the 10 May 1996 event, the AKR intensifications and magnetic field perturbations on the ground all began before dipolarizations were observed by Wind. In these cases, it appears that the dipolarizations observed by Wind were intensifications of existing substorms rather than the onset of new activity. The fact that we only found a good correlation between AKR intensifications and dipolarizations in the magnetotail for the multiple dipolarization series events, which consisted of pseudobreakups and very spatially limited auroral activity, also suggests that many regions of activity across the magnetotail combine to produce a substorm. A similar idea was suggested by Nakamura et al. (1994) to explain why the substorm expansion process can sometimes consist of a number of injections and expansions, each with a time scale comparable to that of a pseudobreakup. Nakamura et al. (1994) proposed that multiple intensification regions associated with localized current diversion could cause a global disturbance by combining to produce the signatures associated with the substorm current wedge. Observations from Geotail, Wind, and GOES 9 during the 18 April 1996 substorm studied by Slavin et al. (1997) also suggest that several overlapping regions of activity in the magnetotail are needed to explain the development of the substorm current wedge.

Figure 13 illustrates the development of the substorm current wedge by the formation of a single region of dipolar magnetic field and high pressure extending from 
geosynchronous orbit to the near-tail due to multiple regions of activity observed at larger radial distances in the magnetotail. The spatial scales of individual flow bursts may be fairly small, as indicated by Angelopoulos et al. $(1994,1996)$ and recent Cluster results (Nakamura et al., 2002b, 2004), but as Fig. 13 illustrates, a large number of flow bursts could combine to produce substorm activity. We propose that the accumulated magnetic flux and pressure gradients resulting from the sum of dipolarization events and flow bursts at different local times may be able to sustain the substorm current wedge for the full duration of the substorm and result in widespread, global auroral activity. The initial activation may still occur near midnight, as indicated by past studies; however, the expansion of the substorm in local time appears to result from overlapping dipolarizations caused by flow bursts from a wide range of local times. This scenario is consistent with the idea that reconnection in the magnetotail is bursty and impulsive in nature (Sergeev et al., 1987). The recovery phase would begin when the region of dipolarized magnetic field expands over the near-Earth neutral line and reconnection ceases, as suggested by Baumjohann et al. (1999). After reconnection ceases, no new earthward flow bursts will be created to contribute to the pressure gradients in the near-tail, so the high pressure region close to Earth within the substorm current wedge will begin to dissipate, allowing for the re-establishment of the cross-tail current and the quiet, tail-like magnetic field structure in the night-side magnetosphere. If there is additional energy input into the magnetotail during the expansion phase, a substorm with multiple expansion phase onsets could be the result. In this model, one or two flow bursts with limited spatial extent would result in a pseudobreakup, rather than a full substorm.

\section{Conclusions}

Wind covered a different region of the near magnetotail in $X$ and $Y$ GSM than earlier studies, but the characteristics of the dipolarization events in the Wind data set are consistent with other studies of magnetic field dipolarizations. Most of the dipolarizations occurred close to the neutral sheet and the spatial distribution of the dipolarization events was peaked in the pre-midnight sector. The total magnetic field and magnitude of $B_{x}$ generally decreased during the isolated dipolarization events. $78 \%$ of the Wind dipolarization events were accompanied by more than $5 \mathrm{~min}$ of earthward flows $>100 \mathrm{~km} / \mathrm{s}$. In $57 \%$ of the Wind dipolarization events, the maximum earthward flow velocities were greater than $300 \mathrm{~km} / \mathrm{s}$. The distribution of multiple dipolarization series events (spaced less than $20 \mathrm{~min}$ apart) was more localized than the distribution of isolated events. The multiple dipolarization series events generally featured increases in $B_{z}$ and the magnetic field inclination. They did not have a significant change in $B_{x}$ or the total magnetic field. Some of the multiple dipolarization series events, such as the 26 July 1997 event, lacked typical growth phase features, such as tail-like magnetic fields and plasma sheet thinning. This may be an indication that some of the multiple dipolarization series events represent a directly driven mode of magnetotail convection, as opposed to the usual substorm sequence of the storage of magnetic energy in the tail and its subsequent release in large bursts of earthward flow.

Even during typical substorm events that began with a period of quiet, tail-like magnetic field and plasma sheet thinning, multiple dipolarizations were often observed by Wind. This may be an indication that even during a typical substorm, dipolarization of the magnetotail is not a sudden, global event, and that substorms can involve both the release of energy stored in the magnetotail and directly driven activity. Instead, the substorm expansion phase appears to be the result of the accumulation of dipolarized flux transported by localized bursts of earthward flow. These localized flow bursts combine to build-up a region with strong pressure gradients in the near-Earth region that is sustained for the duration of the substorm by continued earthward flow bursts. The region of high pressure will be maintained until reconnection and the flows cease, marking the start of the recovery phase. Although the statistical study of Wind data presented here and recent results from Cluster have provided new information on the nature of dipolarizations and their spatial and temporal scales, additional multiple-spacecraft studies are needed to determine how dipolarizations propagate through the magnetotail and to verify whether the global reconfiguration of the magnetotail associated with substorms results from the expansion of a single dipolarization front or is the result of many combined disturbances as described here.

Acknowledgements. This paper has benefitted from discussions with R. Hoffman, J. Gjerloev, D. Gurnett, W. Kurth, and D. Menietti. The CANOPUS array was constructed and is operated by the Canadian Space Agency. K. Sigsbee was a National Research Council Post-doctoral Research Associate at NASA Goddard Space Flight Center while carrying out portions of this research. Work at the University of Iowa was supported by NSF grant ATM-0230647.

Topical Editor T. Pulkkinen thanks T. Nagai and K. Shiokawa for their help in evaluating this paper.

\section{References}

Angelopoulos V., Kennel, C. F., Coroniti, F. V., Pellat, R., Kivelson, M. G., Walker, R. J., Russell, C. T., Baumjohann, W., Feldman, W. C., and Gosling, J. T.: Statistical characteristics of bursty bulk flow events, J. Geophys. Res., 99, 21 257-21 280, 1994.

Angelopoulos, V., Coroniti, F. V., Kennel, C. F., Kivelson, M. G., Walker, R. J., Russell, C. T., McPherron, R. L., Sanchez, E., Meng, C.-I., Baumjohann, W., Reeves, G. D., Belian, R. D., Sato, N., Friis-Christensen, E., Sutcliffe, P. R., Yumoto, K., and Harris, T.: Multipoint analysis of a bursty bulk flow event on 11 April 1985, J. Geophys. Res., 101, 4967-4989, 1996.

Angelopoulos, V., Coroniti, F. V., Kennel, C. F., Kivelson, M. G., Walker, R. J., Russell, C. T., McPherron, R. L., Sanchez, E., Meng, C.-I., Baumjohann, W., Reeves, G. D., Belian, R. D., Sato, N., Friis-Christensen, E., Sutcliffe, P. R., Yumoto, K., and Harris, T.: Correction to "Multipoint analysis of a bursty bulk flow event on 11 April 1985," J. Geophys. Res., 102, 211-212, 1997a. 
Angelopoulos, V., Phan, T. D., Larson, D. E., Mozer, F. S., Lin, R. P., Tsuruda, K., Hayakawa, H., Mukai, T., Kokubun, S., Yamamoto, T., Williams, D. J., McEntire, R. W., Lepping, R. P., Parks, G. K., Brittnacher, M., Germany, G., Spann, J., Singer, H. J., and Yumoto, K.: Magnetotail flow bursts: Association to global magnetospheric circulation, relationship to ionospheric activity, and direct evidence for localization, Geophys. Res. Lett., 24, 2771-2274, 1997b.

Bargatze, L. F., Baker, D. N., McPherron, R. L., and Hones, E. W., Jr.: Magnetospheric impulse response for many levels of geomagnetic activity, J. Geophys. Res., 90, 6387-6394, 1985.

Baumjohann, W., Hesse, M., Kokubun, S., Mukai, T., Nagai, T., and Petrukovich, A. A.: Substorm dipolarization and recovery, J. Geophys. Res., 104, 24 995-25 000, 1999.

Birn, J., Hesse, M., Haerendel, G., Baumjohann, W., and Shiokawa, K.: Flow braking and the substorm current wedge, J. Geophys. Res., 104, 19895-19903, 1999.

Bougeret, J.-L., Kaiser, M. L., Kellogg, P. J., Manning, R., Goetz, K., Monson, S. J., Monge, N., Friel, L., Meetre, C. A., Perche, C., Sitruk, L., and Hoang, S.: WAVES: The Radio and Plasma Wave Investigation on the Wind Spacecraft, Space Sci. Rev., 71, 231-263, 1995.

Fairfield, D. H., Mukai, T., Lui, A. T. Y., Cattell, C. A., Reeves, G. D., Nagai, T., Rostoker, G., Singer, H. J., Kaiser, M. L., Kokubun, S., Lazarus, A. J., Lepping, R. P., Nakamura, M., Steinberg, J. T., Tsuruda, K., Williams, D. J., and Yamamoto, T.: Geotail observations of substorm onset in the inner magnetotail, J. Geophys. Res., 103, 103-117, 1998.

Fairfield, D. H.: A statistical determination of the shape and position of the geomagnetic neutral sheet, J. Geophys. Res., 85, 775-780, 1980 .

Fillingim, M. O., Parks, G. K., Chen, L. J., Brittnacher, M., Germany, G. A., Spann, J. F., Larson, D., and Lin, R. P.: Coincident POLAR/UVI and WIND observations of pseudobreakups, Geophys. Res. Lett., 27, 1379-1382, 2000.

Fillingim, M. O., Parks, G. K., Chen L. J., McCarthy, M., Spann, J. F., and Lin, R. P.: Comparison of plasma sheet dynamics during pseudobreakups and expansive aurorae, Phys. Plasmas, 8, 11271132, 2001

Gallagher, D. L. and Gurnett, D. A.: Auroral kilometric radiation, time-averaged source location, J. Geophys. Res., 84, 6501-6509, 1979.

Green, J. L., Gurnett, D. A., and Shawhan, S. D.: The angular distribution of auroral kilometric radiation, J. Geophys. Res. 82, 1825-1838, 1977.

Gurnett, D. A.: The Earth as a radio source: terrestrial kilometric radiation, J. Geophys. Res., 79, 4227-4238, 1974.

Hesse, M. and Birn, J.,: On dipolarization and its relation to the substorm current wedge, J. Geophys. Res., 96, 19417-19426, 1991.

Kepko, L. and Kivelson, M. G.: Generation of Pi2 pulsations and bursty bulk flows, J. Geophys. Res., 104, 25 021-25 034, 1999.

Kepko, L. and Kivelson, M. G.: Flow bursts, braking, and Pi2 pulsations, J. Geophys. Res., 106, 1903-1915, 2001.

Kokubun, S. and McPherron, R. L.: Substorm signatures at synchronous altitude, J. Geophys. Res., 86, 11 265-11 277, 1981.

Kurth, W. S. and Gurnett, D. A.: Auroral kilometric radiation integrated power flux as a proxy for AE, Adv. Space Res., 22, 73-77, 1998.

Lepping, R. P., Acuña, M., Burlaga, L., Farrell, W., Slavin, J., Schatten, K., Mariani, F., Ness, N., Neubauer, F., Whang, Y. C., Byrnes, J., Kennon, R., Panetta, P., Scheifele, J., and Worley,
E.: The Wind magnetic field investigation, Space Sci. Rev., 71, 207-229, 1995.

Lin, R. P., Anderson, K. A., Ashford, S., Carlson, C., Curtis, D., Ergun, R., Larson, D., McFadden, J., McCarthy, M., Parks, G. K., Rème, H., Bosqued, J. M., Coutelier, J., Cotin, F., DUston, C., Wenzel, K.-P., Sanderson, T. R., Henrion, J., Ronnet, J. C., and Paschmann, G.: A three-dimensional plasma and energetic particle investigation for the Wind spacecraft, Space Sci. Rev., 71, 125-153, 1995.

Lopez, R. E., Lui, A. T. Y., Sibeck, D. G., McEntire, R. W., Zanetti, L. J., Potemra, T. A., and Krimigis, S. M.: The longitudinal and radial distribution of magnetic reconfigurations in the near-Earth magnetotail as observed by AMTE/CCE, J. Geophys. Res., 93, 997-1001, 1988.

Mende, S. B., Frey, H. U., Morsony, B. J., and Immel, T. J.: Statistical behavior of proton and electron auroras during substorms, J. Geophys. Res., 108(A9), 1339, doi:10.1029/2002JA009751, 2003.

Miyashita, Y., Machida, S., Nishida, A., Mukai, T., Saito, Y., and Kokubun, S.: Geotail observations of total pressure and electric field variations in the near and mid-distant tail associated with substorm onsets, Geophys. Res. Lett., 26, 639-642, 1999.

Murata, T., Matsumoto, H., Kojima, H., and Iyemori, T.: Correlations of AKR index with $K_{p}$ and $D_{s t}$ indices, in Proc. NIPR Symp. Upper Atmos. Phys. 10, 64-68, 1997.

Nagai, T.: An empirical model of substorm-related magnetic field variations at synchronous orbit, in Magnetospheric Substorms, Geophysical Monograph 64, edited by Kan, J. R., Potemra, A., Kokubun, S., and Iijima T., 91-95, American Geophysical Union, Washington, D.C., 1991.

Nagai, T.: Observed magnetic substorm signatures at synchronous altitude, J. Geophys. Res., 87, 4405-4417, 1982.

Nagai, T., Singer, H. J., Mukai, T., Yamamoto, T., and Kokubun, S.: Development of substorms in the near-Earth tail, Adv. Space Res., 25(7/8), 1651-1662, 2000.

Nagai, T., Baker, D. N., and Higbie, P. R.: Development of substorm activity in multiple-onset substorms at synchronous orbit, J. Geophys. Res., 88, 6994-7004, 1983.

Nakai, H. and Kamide, Y.: A critical condition in magnetotail pressure for leading to a substorm expansion onset: Geotails observations, J. Geophys. Res., 109(A01205), doi:10.1029/2003JA010070, 2004.

Nakamura, R., Baumjohann, W., Mouikis, C., Kistler, L. M., Runov, A., Volwerk, M., Asano, Y., Voros, Z., Zhang, T. L., Klecker, B., Rème, H., and Balogh, A.: Spatial scale of high-speed flows in the plasma sheet observed by Cluster, Geophys. Res. Lett., 31(L09804), doi:10.1029/2004GL019558, 2004.

Nakamura, R., Baumjohann, Runov, A., Volwerk, M., Zhang, T. L., Klecker, B., Bogdanova, Y., Roux, A., Balogh, A., Rème, H., Sauvaud, J. A., and Frey, H. U.: Fast flow during current sheet thinning, Geophys. Res. Lett., 29(23), doi:1029/2002GL016200, 2002a.

Nakamura, R., Baumjohann, W., Klecker, B., Bogdanova, Y., Balogh, A., Rème, H., Bosqued, J. M., Dandouras, I., Sauvaud, J. A., Glassmeier, K.-H., Kistler, L., Mouikis, C., Zhang, T. L., Eichelberger, H., and Runov, A.: Motion of the dipolarization front during a flow burst event observed by Cluster, Geophys. Res. Lett., 29(20), 1942, doi:10.1029/2002GL015763, 2002 b.

Nakamura, R., Baker, D. N., Yamamoto, T., Belian, R. D., Bering, E. A., Benbrook, J. R., and Theall, J. R.: Particle and field signatures during pseudobreakup and major expansion onset, J. Geophys. Res., 99, 207-222, 1994. 
Raj, A., Phan, T., Lin, R. P., and Angelopoulos, V.: Wind survey of high-speed bulk flows and field-aligned beams in the near-Earth plasma sheet, J. Geophys. Res., 107(A12), 1419, doi:10.1029/2001JA007547, 2002.

Sergeev, V. A., Pellinen, R. J., and Pulkkinen, T. I.: Steady magnetospheric convection: A review of recent results, Space Sci. Rev., 75, 551-604, 1996.

Sergeev, V. A., Semenov, V. S., and Sidneva, M. V.: Impulsive reconnection in the magnetotail during substorm expansion, Planet. Space Sci., 35, 1199-1212, 1987.

Shiokawa, K., Baumjohann, W., and Haerendel, G.: Braking of high-speed flows in the near-Earth tail, Geophys. Res. Lett., 24, 1179-1182, 1997.

Shiokawa, K., Baumjohann, W., Haerendel, G., Paschmann, G., Fennell, J. F., Friis-Christensen, E., Lühr, H., Reeves, G. D., Russell, C. T., Sutcliffe, P. R., and Takahashi, K.: High-speed ion flow, substorm current wedge, and multiple $\mathrm{Pi} 2$ pulsations, J. Geophys. Res., 103, 4491-4507, 1998.

Sigsbee, K., Cattell, C. A., Fairfield, D., Tsuruda, K., and Kokubun, S.: Geotail observations of low- frequency waves and high-speed earthward flows during substorm onsets in the near magnetotail from 10 to $13 R_{E}$, J. Geophys. Res., 107(A7), doi10.1029/2001JA000166, 2002.
Sigsbee, K., Slavin, J. A., and Øieroset, M.: Magnetotail convection during substorms and directly driven events, in Substorms-7: Proceedings of the 7th International Conference on Substorms, 27-30, edited by T. Pulkkinen and N. Ganushkina, Finnish Meteorological Institute, Helsinki, Finland, 2004.

Singer, H. J., Hughes, J. W., Fougere, P. F., and Knecht, D. J.: The localization of Pi 2 pulsations: ground-satellite observations, J. Geophys. Res., 88, 7029-7036, 1983.

Slavin, J. A., Fairfield, D. H., Lepping, R. P., Szabo, A., Reiner, M. J., Kaiser, M., Owen, C. J., Phan, T., Lin, R., Kokubun, S., Mukai, T., Yamamoto, T., Singer, H. J., Romanov, S. A., Buechner, J., Iyemori, T., and Rostoker, G.: Wind, Geotail, and GOES 9 observations of magnetic field dipolarization and bursty bulk flows in the near-tail, Geophys. Res. Lett., 24, 971-974, 1997.

Slavin, J. A., Fairfield, D. H., Lepping, R. P., Hesse, M., Ieda, A., Tanskanen, E., Østgaard, N., Mukai, T., Nagai, T., Singer, H. J., and Sutcliffe, P. R.: Simultaneous observations of earthward flow bursts and plasmoid ejection during magnetospheric substorms, J. Geophys. Res., 107(A7), doi:10.1029/2000JA003501, 2002. 